\title{
Molecular basis for the R-type anion channel QUAC1 activity in guard cells
}

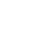

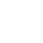

Li Qin ${ }^{1,2 \#}$, Ling-hui Tang ${ }^{1,2 \#}$, Jia-shu Xü ${ }^{3,4 \#}$, Xian-hui Zhang ${ }^{1,2 \#}$, Y un Zhu ${ }^{3,4 \#}$, Chun-rui Zhang $^{1,2}$, Xue-lei Liu ${ }^{1,2}$, Mei-hua Wang ${ }^{1,2}$, Fei Li $^{5}$, Fei Sun $^{3,4}$, Min Su$^{1,2^{*}}$, Yu-jia Zhai ${ }^{3,4^{*}}$ \&

Yu-hang Chen ${ }^{1,2^{*}}$

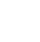

${ }^{1}$ State Key Laboratory of Molecular Developmental Biology, Institute of Genetics and Developmental Biology, Chinese Academy of Sciences, Beijing, China; Innovative Academy of Seed Design, Chinese Academy of Sciences, Beijing 100101, China

${ }^{2}$ College of Advanced Agricultural Sciences, University of Chinese Academy of Sciences, Beijing 100049, China

3 National Laboratory of Biomacromolecules, CAS Center for Excellence in Biomacromolecules, Institute of Biophysics, Chinese Academy of Sciences, Beijing 100101, China

${ }^{4}$ School of Life Sciences, University of Chinese Academy of Sciences, Beijing 100049, China ${ }^{5}$ Department of Biology, New York University, New York, NY 10003, USA

${ }^{\#}$ These authors contributed equally to this work.

${ }^{*}$ Correspondence to:

2 Yu-hang Chen, E-mail: yuhang.chen@genetics.ac.cn; or Yu-jia Zhai, E-mail: yujia@ibp.ac.cn; or Min Su, E-mail: minsu@genetics.ac.cn. 


\section{SUMMARY}

27 The rapid (R)-type anion channel plays a central role in controlling stomatal closure in plant guard cells, thus regulating the exchange of water and photosynthetic gas $\left(\mathrm{CO}_{2}\right)$ in response to environmental stimuli. The activity of the R- type anion channel is regulated by malate. However, the molecular basis of the R-type anion channel activity remains elusive. Here, we

31 describe the first cryo-EM structure of the R-type anion channel QUAC1 at $3.5 \AA$ resolution in 32 the presence of malate. The structure reveals that the QUAC1 is a symmetrical dimer, forming 33 a single electropositive T-shaped pore for passing anions across the membrane. The 34 transmembrane and cytoplasmic domains are assembled into a twisted bi-layer architecture, with the associated dimeric interfaces nearly perpendicular. Our structural and functional analyses reveal that QUAC1 functions as an inward rectifying anion channel and suggests a mechanism for malate-mediated channel activation. Altogether, our study uncovers the molecular basis for a novel class of anion channels and provides insights into the gating and modulation of the R-type anion channel.

\section{KEYWORDS}




\section{INTRODUCTION}

Stomatal pore, formed by a pair of guard cells in leaf epidermis, plays a crucial role in regulating $\mathrm{CO}_{2}$ assimilation and water evaporation in plants ${ }^{1,2}$. Guard cells can integrate a wide range of environmental stimuli, such as drought, humidity, high $\mathrm{CO}_{2}$, and ozone, and convert them into appropriate turgor pressure changes that regulate stomatal opening or closure ${ }^{3,4}$.

It is well-established that anion effluxes are key events to initiate stomatal closure in response to environmental stimuli ${ }^{5-8}$. Previous studies showed that the guard cells harbour two distinct types of anion channels: $\operatorname{rapid}(\mathrm{R})$-type and slow (S)-type ${ }^{9-12}$. The R-type anion channel can be fully activated and rapidly inactivated within milliseconds, whereas the S-type anion channel takes up to several tens of seconds to be activated, followed by slow inactivation

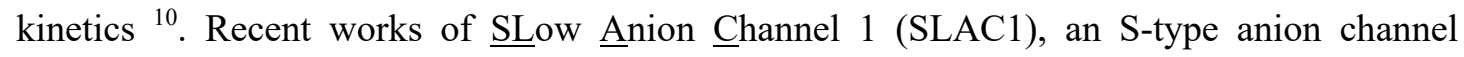
discovered in Arabidopsis ${ }^{13-15}$, have uncovered detailed mechanisms underlying S-type anion channel activity ${ }^{16-21}$. However, the molecular basis of the biological role of the R-type anion channel is still largely enigmatic due to the lack of structural information.

QUick Anion Channel 1 (QUAC1), initially named aluminium-activated malate transporter12 (ALMT12), represents a guard cell R-type anion channel in Arabidopsis ${ }^{22}$. The loss-of-function of quac1 (almt12) mutation resulted in impaired stomatal closure ${ }^{22,23}$. A double mutant lacking SLAC1 and QUAC1 was nearly unresponsive to various stimuli that were expected to cause stomatal closure ${ }^{24}$. Apart from its role in stomatal regulation, QUAC1 also plays a vital role in cell polarity and the growth of pollen tubes via regulation of apical anion efflux ${ }^{25,26}$. The activity of QUAC1 was reported to be modulated by malate ${ }^{22,27-29}$, OST1 phosphorylation ${ }^{30}$ and calmodulin ${ }^{31}$, but the underlying mechanisms remain elusive.

Here, we report the first cryo-EM structure of soybean QUAC1 from Glycine max ( GmQUAC1, $\sim 67 \%$ sequence identity to Arabidopsis AtQUAC1) at the resolution of $3.5 \AA$ in the presence of malate. The structure revealed that the QUAC1 is a symmetrical dimer assembled into twisted bi-layer architecture with a central T-shaped pore across the membrane. The pore is lined with highly conserved positively charged residues, rendering the pore surface electropositive, consistent with the QUAC1 function as an anion channel. Further 
electrophysiological analyses reveal that the QUAC1 displays rapid kinetics in channel gating during activation and inactivation.

Our truncation study reveals that the N-terminal juxtamembrane helix is crucial in maintaining structural integrity for proper channel function. Further mutagenesis analyses demonstrate that the $\mathrm{C}$-terminal interaction locks the channel in a high-energy state, and that a domain-swapped helix plays an inhibitory role in channel regulation. Two pore-lining W90 residues, close to the lateral fenestration within the transmembrane domain, serve as toggle switches to regulate gating during channel activation. Therefore, the unique architecture endows QUAC1 function as an R-type anion channel, and our structural and functional analyses allow us to propose a novel mechanism for malate-mediated channel activation. Altogether, our study uncovers the molecular basis for a novel class of anion channels and provides insights into the gating and modulation of the R-type anion channel.

\section{RESULTS}

\section{Bioinformatics analyses of plant ALMTs/QUACs}

QUAC1 belongs to the ALMT family, which has diverse functions in plants, including stomatal function ${ }^{22,23,32-34}$, pollen tube growth ${ }^{25,26}, \mathrm{Al}^{3+}$ resistance ${ }^{35-38}$, mineral nutrition ${ }^{39,40}$, fruit acidity ${ }^{41,42}$, microbe interactions ${ }^{43,44}$, and seed development ${ }^{45,46}$. There are thirteen members in the model plant Arabidopsis thaliana, except for one short sequence of a partial transmembrane domain (ALMT11). To better understand how QUAC (ALMT) proteins are represented in the plant kingdom, we clustered $\sim 1,700$ non-redundant sequences into a superfamily at the PSI-BLAST level $\mathrm{E} \leq 5 \times 10^{-3}$, then into two distinct families at a threshold of $\mathrm{E} \leq 10^{-160}$, and finally into subfamilies at a threshold of $\mathrm{E} \leq 10^{-180}$, as detailed in Extended Data Table 1.

Since more studies have shown that ALMT members function as anion channels rather than transporters, and the QUAC1 is now the best-characterized member, we adopted nomenclature for the QUAC superfamily, which was further divided into families QF1 and QF2.

We further found that family QF1 has two subfamilies: QF1A comprises the Arabidopsis ALMT3, 4, 5, 6, 9 and their homologs, and QF1B has a distinct set of ALMT proteins. Family 
111 QF2 has three subfamilies: the Arabidopsis ALMT1, 2, 7, 8, 10 proteins are in subfamily QF2A,

112 the Arabidopsis ALMT12, 13, 14 proteins are in QF2B, and another distinct set of ALMT 113 proteins are in QF2C (Extended Data Fig. 1).

114 The structure-based sequence alignment for the GmQUAC1 and thirteen Arabidopsis 115 QUAC/ALMTs is shown in Extended Data Fig. 2. Sequence analyses reveal that the ALMTs 116 share relatively conserved transmembrane and cytosolic helical regions. Some conserved 117 positively charged residues are distributed in the transmembrane regions and may be 118 responsible for anion selectivity or voltage sensing. Some fingerprint motifs, including WEP 119 (Trp-Glu-Pro) and PXWXG (Pro-X-Trp-X-Gly), are found in the cytosolic helical region. One 120 of the most divergent regions ( $~ 50-100$ aa in length) is found between $\mathrm{H} 5$ and H6 helices in the 121 cytosolic portions, harbouring potential phosphorylation residues (Ser/Thr) for channel 122 modulation. Further studies will provide mechanistic insight into the regulation of these 123 characteristic motifs or distinct sets of QUACs.

\section{Cryo-EM structural determination of QUAC1}

126 We screened six plant QUAC1s for expression and found that the GmQUAC1 was suitable for 127 further structural and functional studies (see method for details). Upon solubilization in 1.0\% $128 \mathrm{n}$-dodecyl- $\beta$-D-maltopyranoside (DDM) and $0.02 \%$ cholesteryl hemisuccinate (CHS), the $129 \mathrm{Gm} \mathrm{QUAC1}$ proteins were purified by $\mathrm{Ni}^{2+}$-affinity chromatography, and the resulting peak130 fractions were pooled and loaded onto a gel-filtration column for further purification, buffer 131 and detergent exchange. The final solution contained $0.005 \%$ detergent LMNG, with either 150 $\mathrm{mM} \mathrm{NaCl}$ or $75 \mathrm{mM}$ L-malate.

133 We further determined its cryo-EM structure in the presence of $75 \mathrm{mM}$ L-malate 134 (Extended Data Fig. 3, 4 and Extended Data Table 2). The dimeric reconstruction at $3.5 \AA$ 135 resolution allowed de novo modelling of 410 of the 537 amino acids (residues 36-393 and 455136 506) per protomer chain. Other regions are not resolved in the density map due to their intrinsic

137 flexibility in the protein. The search in the Dali server ${ }^{47}$ for similar structures returned no 138 significant hit. To the best of our knowledge, the QUAC1 protomer has a unique protein fold 139 and architecture, representing a novel class of ion channels. 


\section{The QUAC1 channel forms a twisted bi-layer architecture}

142 The QUAC1 channel is a flat vase-shaped homodimer with a two-fold axis perpendicular to the

143 plasma membrane (Fig. 1a and Supplementary Video 1). The overall molecule forms a bi-layer

144 architecture, divided into two portions of the transmembrane domain (TMD) and the 145 cytoplasmic helical domain (CHD). The domain topology for each protomer is shown in Fig.

146 1b. The TMD comprises 6 TM helices, arranging as three pairs of V-shaped helical hairpins 147 stacked against each other, whereas the CHD has 7 helices, forming a helical bundle with a 148 short domain-swapped finger helix. The N-terminal pre-TM region contains a juxtamembrane 149 helix bent at a conserved proline before the TMD. A disorder region between helix H5 and H6 150 in the CHD enriches with Ser/Thr residues (Extended Data Fig. 2), raising an intriguing 151 possibility of containing phosphorylation sites for channel regulation.

152 The dimer formation is mediated by interactions from both TMDs and CHDs, which bury 153 surface areas of $\sim 3,500 \AA^{2}$ and $\sim 3,300 \AA^{2}$, respectively. When viewed from the extracellular 154 side, the TM helices trace the circumference of an ellipse with TM1-TM6 from one protomer 155 followed by another set of TM1-TM6 from the second protomer in an antiparallel manner (Fig. 156 1c). At the membrane, TM1 and TM2 directly interact with TM4 and TM5 from another 157 protomer, respectively. In the cytoplasm, the CHD of each protomer unites together at the distal 158 end of the molecule, mediated by intensive interactions. A domain-swapped finger helix (H6) 159 also participates in C-terminal interactions (Fig. 1d). However, the functional role of these 160 interactions remains unclear.

161 In the bi-layer structure of QUAC1, the TMDs connect to the CHDs via a highly conserved 162 PXWXG motif (Pro-X-Trp-X-Gly). They interact with each other in a twisted manner, with 163 their dimeric interfaces nearly perpendicular to each other (forming a dihedral angle of $\sim 83^{\circ}$ ) 164 (Fig. 2a). Interestingly, another characteristic motif WEP (Trp-Glu-Pro), located in the 165 connecting loop between helix $\mathrm{H} 2$ and $\mathrm{H} 3$, is found in the vicinity of the PXWXG motif at the 166 layer interface between TMD and CHD. These two motifs, together with other surrounding 167 charged residues (Arg/Lys: R56, K63, R291, and R294; Asp/Glu: D54, E223, D224, and E289), 
168 form intensive interactions at the layer interface, and thus may play a vital role in coupling

169 channel regulation to gating (Fig. 2b). Mutations in the WEP motif (E286Q in TaALMT1 and

170 E276Q in AtQUAC1) ${ }^{27,48}$ completely abolish their channel activities, possibly due to impaired

171 coupling between the TMD and CHD caused by the altered interaction at the layer interface.

172 Thus, the unique architecture design causes the channel in a high-energy state, providing a

173 structural basis for QUAC1 function as an R-type channel. These observations also imply that

174 the intramolecular domain rearrangements may occur upon channel activation.

175

176 Structural features of the ion conduction pathway in QUAC1

177 Unlike the trimeric SLAC1, which has three independent pores ${ }^{20}$, the dimeric QUAC1 forms

178 a single T-shaped tunnel with a bifurcated entrance in the cytoplasm (Fig. 3a). The TM2, TM3,

179 TM5, and TM6 from both protomers create a pore with a radius of $\sim 4-6 \AA$ within the membrane

180 (Fig. 3b), as estimated by HOLE ${ }^{49}$. Overall, the QUAC1 pore is lined with highly conserved

181 and general hydrophobic residues (Fig. 3c). Nevertheless, some highly conserved positively

182 charged residues are distributed along the pore, thus rendering the surface electropositive (Fig.

$1833 d)$

184 Two arginines (R187 and R198) form a positively charged ring facing the pore entrance

185 at the extracellular side. Three positively charged residues from TM3 and TM4 (K109, R113,

186 and R158) protrude into the pore, making a second positively charged ring within the membrane.

187 They are associated with other conserved residues from TM2 and TM5 (E100, D168, and Y169),

188 mediating an inter-helices network within the pore. Another two lysines (K164 and K165) form

189 additional constrictions at the bottom of the T-shaped pore (Fig. 3e). Altogether, the structural

190 feature of QUAC1 likely contributes to its function as an anion-conducting channel. The above

191 findings suggest that permeable anions interact with the pore-lining charged residues, thus

192 participating in channel regulation.

193 Remarkably, a central kink $\left(\sim 24^{\circ}\right)$ in TM6 causes a lateral fenestration from the membrane

194 to the lumen of the channel pore within each protomer (Fig. 4a). The fenestration measures $\sim 6$

$195 \AA \times 20 \AA$, and its dimension depends upon the conformation of pore-forming TMs, especially 
the kinked TM6. The presence of kinked helix and lateral fenestration in the structure implies

197 that the channel in $75 \mathrm{mM}$ malate is in a constrained conformation. Interestingly, several

198 unmodeled densities and a highly conserved pore-lining W90 residue are found within or near

199 the fenestration (Fig. 4b), raising an interesting question if the bulky W90 residue serves as a 200 toggle switch to regulate channel gating during QUAC1 activation.

\section{Functional characterization of QUAC1 by TEVC}

203 A total of 34 ALMTs were found in soybean ${ }^{50}$, but none of them has yet been functionally 204 characterized by electrophysiology. We expressed the GmQUAC1/ALMT12 in Xenopus laevis 205 oocytes and measured channel conductance by applying the two-electrode voltage-clamp 206 (TEVC) technique. In the recordings, the application of voltage pulses elicited rapidly 207 activating currents in the bath solution of malate, resembling the AtQUAC1 ${ }^{22}$. We also found 208 that the steady-state currents mediated by GmQUAC1 displayed a bell-shaped current-voltage 209 curve (Extended Data Fig. 5). This electrophysiological behaviour points to a strong voltage 210 dependence for the QUAC1 channel activation, a hallmark feature of the R-type anion channel 211 in guard cells ${ }^{10,22}$.

212 We also measured the conductance of GmQUAC1 in other bath solutions. Surprisingly, 213 we found that the activation/inactivation kinetics and voltage-dependency of GmQUAC1 in $214 \mathrm{NaNO}_{3}$ or $\mathrm{NaCl}$ solutions are distinguished from those in malate solution (Extended Data Fig.

215 5). In contrast to substantial instantaneous currents, only small steady-state currents can be seen 216 in the recording of $G m \mathrm{QUAC1}$ in the bath solution of $\mathrm{NaNO}_{3}$ or $\mathrm{NaCl}$ (Extended Data Fig. 5).

217 Overall, our TEVC recordings demonstrate that external anions play a crucial role in regulating 218 QUAC1 channel properties, especially the activation/inactivation kinetics and steady-state 219 currents. We hypothesize that the anions interplay with the pore-lining residues, such as the 220 inter-helices charged residues interacting network within the pore (Fig. 3e), and act as door 221 stopper wedges to regulate channel gating. 


\section{Single-channel analysis of QUAC1 channel in planar lipid bilayer}

225 To further understand the single-channel properties, we first fused the $G m \mathrm{QUAC1}{ }^{\mathrm{NaCl}}$ proteins 226 (purified in $150 \mathrm{mM} \mathrm{NaCl}$ ) into a lipid bilayer and measured channel conductance under 227 different symmetrical solutions (Fig. 5a). When using symmetrical $\mathrm{NaNO}_{3}$ solutions, the 228 application of a series of transmembrane voltages to the trans chamber resulted in frequent 229 openings and closings of the GmQUAC1 channel, as evidenced by the current fluctuation in 230 the recording traces at different applied voltages (Extended Data Fig. 6a). We also found that 231 the channel gating transits between two predominant full open and closed states, and sometimes 232 to a sub-conductance that is $\sim 50 \%$ level of the full conductance (Fig. 5b). This observation is 233 consistent with the two-entrance T-shaped tunnel presented in the QUAC1 channel (Fig. 3a). 234 At $\sim 50 \%$ of the full level, the intermediate sub-conductance suggested one of two entrances 235 was blocked or closed. In addition, the QUAC1 channel gating displays a flickering feature that 236 represents the rapid character of the activation/inaction kinetics for R-type anion channels.

237 In contrast, we observed much reduced channel activity in the measurements in the 238 solution of $150 \mathrm{mM} \mathrm{NaCl}$ (Extended Data Fig. 6b). The mean single-channel conductance for 239 the $G m \mathrm{QUAC}^{\mathrm{NaCl}}$ is $75.5 \pm 4.5 \mathrm{pS}(\mathrm{n}=5)$ and $58.5 \pm 7.0 \mathrm{pS}(\mathrm{n}=5)$ in the solutions of $\mathrm{NaNO}_{3}$ 240 and $\mathrm{NaCl}$, respectively (Fig. 5c). Intriguingly, the channel open probability displays a strong 241 voltage dependency, much higher at positive voltage than at negative one (Fig. 5d). As in our 242 experimental setup, positive currents mean anions flow from the cis (representing cytoplasm) 243 to the trans-chamber (representing extracellular side), and vice versa. Therefore, these results 244 demonstrate that the QUAC1 channel functions as an R-type anion channel, favouring efflux 245 (corresponding to anions flow from cis- to trans-chamber).

247 Malate regulates QUAC1 channel gating.

248 To investigate the effect of malate on the channel activity, we reproduced the same experiments 249 with the $G m \mathrm{QUAC1}^{\text {malate }}$ (purified in $75 \mathrm{mM} \mathrm{L-malate)} \mathrm{as} \mathrm{above.} \mathrm{Interestingly,} \mathrm{we} \mathrm{observed}$ 250 that $G m \mathrm{QUAC} 1^{\text {malate }}$ exhibits a similar current amplitude but a much higher open probability 251 than those with $\mathrm{GmQUAC1}{ }^{\mathrm{NaCl}}$ in both solutions of $\mathrm{NaNO}_{3}$ and $\mathrm{NaCl}$ (Fig. 5c and Extended 
252 Data Fig. 6). The mean single-channel conductance for the $G m \mathrm{QUAC}^{\text {malate }}$ is $88.5 \pm 2.4 \mathrm{pS}$ $253(\mathrm{n}=4)$ and $62.7 \pm 4.1 \mathrm{pS}(\mathrm{n}=4)$ in the solutions of $\mathrm{NaNO}_{3}$ and $\mathrm{NaCl}$, respectively. The open 254 probabilities of $G m \mathrm{QUAC} 1^{\text {malate }}$ also display a similar voltage dependency as observed for $255 G m \mathrm{QUAC1}^{\mathrm{NaCl}}$ (Figure 5D and S6). Taken together, our results suggest that malate regulates 256 QUAC1 activation by modulating channel opening probability.

257 To further examine malate regulation on the channel gating, $2 \mathrm{mM} \mathrm{L-malate}$ was added to 258 the solutions upon the $G m \mathrm{QUAC} 1^{\mathrm{NaCl}}$ fused into a lipid bilayer. Interestingly, the channels 259 became more active after adding $2 \mathrm{mM} \mathrm{L}$-malate to either the trans- or cis-chamber (Fig. $6 \mathrm{a}$ 260 and 6b). The current amplitude slightly increases, whereas the open probability becomes much 261 higher (Fig. 6c and 6d). These observations confirm that malate can stimulate channel activity 262 via enhanced open probability. Our findings also suggest that the malate regulation site may be 263 located within the pore and can be accessed from either the extracellular or intracellular side of 264 the membrane. We speculate that the GmQUAC1 structure in the presence of $75 \mathrm{mM} \mathrm{L}$-malate may represent an open state, although the malate-binding site remained unclear due to 266 insufficient information.

\section{The N-terminal pre-TM helix is required for channel activity.}

269 The sequence analysis shows that the N-terminal segment before TMD is one of the most 270 divergent regions in the QUAC family (Extended Data Fig. 2). The QUAC1 structure reveals 271 that this region contains a juxtamembrane pre-TM helix (Fig. 1b), enriching positively charged residues. To examine its functional role, we designed a truncation construct ( $\Delta 1-53)$.

273 Surprisingly, we found that the $\Delta 1-53$ mutant resulted in nearly null currents in the TEVC 274 recordings (Extended Data Fig. 7). The observation demonstrates that the pre-TM helix is 275 indispensable for channel activity. Considering its association with the inner leaflet of the 276 membrane, we propose that the pre-TM helix may serve as a lever to regulate the proper 277 conformation of the QUAC1 channel. 
The C-terminal dimeric interaction is crucial for channel function.

281 The cytosolic C-terminal portion is mainly formed by $\alpha$-helices, except that a large divergent

282 region between helix $\mathrm{H} 5$ and H6 is disordered or missing in the QUAC family (Extended Data

283 Fig. 2). The GmQUAC1 structure revealed extensive interactions between two CHDs at the 284 distal end of the dimer (Fig. 7a). Two highly conserved hydrophobic residues (F470 and L474 285 from helix H7) protrude into the hydrophobic pocket formed by another protomer. Together 286 with their antiparallel counterparts from another protomer, these bulky hydrophobic residues 287 form a zipper-like interaction at the dimeric interface (Fig. 7b). In addition, a short finger helix 288 (H6), whose sequence is conserved in the subfamily QF2B (Extended Data Fig. 2), also 289 participates in the dimeric interaction in a domain-swapped manner (Fig. 7c). These interactions 290 unite the two CHDs together and thus creates an anchor point for mechanical force transduction 291 between TMD and CHD.

292 To investigate the functional role of the dimeric interactions in channel regulation, we generated mutations, aiming to disrupt the dimer-interface (A477E and F470A/L474A), or to 294 break the finger helix interaction in H6 ( $\triangle 460-467$ and S461D), in both the CHD (residues 221295 537) bacteria-expression constructs for gel-filtration analysis and the full-length oocyte296 expression constructs for conductance measurement. In contrast to the wild type, both dimer297 interface disrupting mutants exhibited backwards-shifted elution peaks (from $9.2 \mathrm{ml}$ to $10.2 \mathrm{ml}$ ) 298 in the gel filtration profile and showed almost null currents in TEVC recording. In another 299 experiment, the two altered helix H6 mutants cause loosened dimers but unexpectedly enhanced 300 channel activities (Fig. 7d-f and Extended Data Fig. 8). Altogether, our results show that the C301 terminal interaction has two important functions: (1) joins the two CHD together and locks the 302 channel in a high-energy state; (2) a domain-swapped helix (H6) plays an inhibitory role, and 303 its release enhances the channel activity. Our results demonstrated that the C-terminal 304 interaction provides a basis for fast regulation of the R-type anion channel. 


\section{Gating of the QUAC1 channel}

308 Our electrophysiological experiments have demonstrated that malate plays a vital role in 309 modulating GmQUAC1 activity; however, we wonder how malate regulation is coupled to 310 channel activation. The GmQUAC1 structure reveals that the conserved pore-lining tryptophan 311 W90 is in close contact with an unmodelled density within the fenestration (Fig. 4b). To 312 understand the functional role of W90, we made mutations of W90A and W90F. Compared to 313 wild-type, the removal of indole ring in W90A mutant causes $\sim 5$ times larger conductance in 314 the presence of external malate. A similar aromatic amino acid substitution in the W90F mutant 315 can stimulate channel currents even larger (Extended Data Fig. 9a and 9b). These observations 316 imply that the conserved W90 may interact with surrounding residues and function as a toggle 317 switch to regulate channel gating.

318 This notion is confirmed in the measurement of combined mutants of W90F with other 319 two loss-of-function mutants, $\Delta 1-53$ and A477E. We observed that both null mutants restore 320 channel activity to a moderate level after the addition of W90F (Extended Data Fig. 9c and 9d). 321 We speculate that malate promotes QUAC1 activation probably via regulating the 322 conformation of tryptophan W90. Distinct from the S-type SLAC1 regulation controlled by 323 phosphorylation/ dephosphorylation ${ }^{16-20}$, our study reveals that the R-type QUAC1 gating can 324 be directly mediated by malate, which is much faster. These findings may explain the fast 325 kinetics observed in the R-type channel, compared with the slow activity found in the S-type 326 channel.

\section{DISCUSSION}

329 Recently, AlphaFold has made significant progress in 3D structure prediction from protein

330 sequence ${ }^{51}$. By taking advantage of this advanced technology, we generated an AlphaFold 331 predicted model for the GmQUAC1 protein that is the focus of our current study. Surprisingly, 332 the AlphaFold predicted GmQUAC1 structure (AF-structure) is surprisingly similar to that 333 determined by cryo-EM (cryo-EM structure), with an r.m.s.d. of $2.1 \AA / 160 \mathrm{C}_{\alpha}$ and $1.4 \AA / 207$ $334 \mathrm{C}_{\alpha}$ for the TMD and CHD, respectively. However, further superimposition reveals a noticeable 
335 difference in the orientation of TMD vs CHD in QUAC1, with an r.m.s.d. of $3.1 \AA / 388 \mathrm{C}_{\alpha}$ and

$3363.2 \AA / 776 \mathrm{C}_{\alpha}$ for the monomer and dimer, respectively (Extended Data Fig. 10a).

337 Unlike the kinked TM6 observed in the cryo-EM structure, the TM6 in the AF-structure 338 adopts a straight conformation, eliminating the lateral fenestration in TMD (Extended Data Fig.

339 10b). As the malate regulation effect on the channel is not taken into account, the resulting 340 predicted model may represent an apo-structure in a low energy state. The cryo-EM structure 341 determined in the presence of malate reveals a high-energy twisted conformation, thus 342 representing a malate-activated structure. These two structures allow us to make comparisons 343 and gain insight into the gating mechanism of the QUAC1 channel. It seems that the pore344 forming helices move slightly outward, as suggested by the porcupine plot on the C $\alpha$ 345 comparison between the AF-structure and the cryo-EM structure (Extended Data Fig. 10c). 346 Another noticeable difference is the dihedral angle between the TMD and CHD interfaces, 347 which is $86.5^{\circ}$ and $83.0^{\circ}$ for the AF-structure and cryo-EM structure, respectively (Extended 348 Data Fig. 10d). This observation shows a slight rotation $\left(\sim 3.5^{\circ}\right)$ between these two states, 349 suggesting that domain reorganization occurs during the conformational conversion. We 350 speculate that the domain reorganization is associated with malate regulation, and subsequently 351 triggers conformational changes of the toggle switch in the pore to activate the channel 352 (Extended Data Fig. 10e and and Supplementary Video 1).

353 Our work provides the first glimpse of the molecular structures of the R-type anion channel. 354 Our study showed that the TMD and CHD portions in QUAC1 interact in a twisted manner, 355 rendering the channel in a high-energy state. Based on our data, we propose the following 356 mechanism for QUAC1 activation: upon malate binding, the QUAC1 undergoes intramolecular 357 domain reorganization to form a twisted bi-layer architecture, which subsequently induces the 358 conformational change of the toggle switch W90. These changes promote the channel 359 conversion from the basal to the activated state. The malate-mediated activation is further 360 enhanced by other modulations, such as kinase phosphorylation or calmodulin-binding, 361 probably via releasing its inhibitory domain-swapped helix from the inter-protomer interaction 362 (Fig. 7g and Supplementary Video 2). Our study provides mechanistic insights into the gating 
363 activation of the R-type channel and offers a plausible explanation for fast kinetics in the R-

364 type currents.

365 As both SLAC1 and QUAC1 represent two essential distinct channels that mediate

366 osmotic active anions efflux from guard cell, one remaining puzzle is how they interplay with

367 each other to initiate stomatal closure in response to various environmental stimuli. Further

368 investigation to address this issue will shed light on the understanding of guard cell stomatal

369 signaling and provide critical information for engineering drought-resistant or water-use

370 efficiency crops.

371 


\section{ACKNOWLEDGMENTS}

376 We thank the staff at the Center for Biological Imaging (CBI), Institute of Biophysics, Chinese

377 Academy of Sciences. This project is financially supported by the National Key Research and 378 Development Program of China (2020YFA0509903 and 2016YFA0500503 to Y.-h.C., and 379 2017YFA0504703 to Y.-j.Z.), the Strategic Priority Research Program of the Chinese Academy 380 of Sciences (XDA24020305 to Y.-h.C., and XDB37040102 to F.S.), and the National Natural 381 Science Foundation of China (31872721 to Y.-h.C., and 31771566 to Y.-j.Z.).

\section{DECLARATION OF INTERESTS}

384 The authors declare no competing financial interests.

\section{AUTHOR CONTRIBUTIONS}

386 L.Q. performed protein purification, Cryo-EM data collection, TEVC experiments, and data 387 analysis; L.-h.T., J.-s.X., Y.Z. performed Cryo-EM data collection and structural determination; 388 X.-h.Z. performed mutagenesis and protein purification; M.S. performed PLB experiments and 389 data analysis; C.-r.Z., X.-1.L., M.-h.W, performed experiments; F.L., F.S., Y.-j.Z. analyzed data; 390 Y.-h.C. initiated the project, planned and analyzed experiments, supervised the research and 391 wrote the manuscript with input from all authors.

\section{REFERENCES}

394 1. Hetherington, A \& Woodward, F. The role of stomata in sensing and driving environmental change.

$395 \quad$ Nature 424, 901-908 (2003).

396 2. Sirichandra, C., Wasilewska, A., Vlad, F., Valon, C. \& Leung, J. The guard cell as a single-cell model 397 towards understanding drought tolerance and abscisic acid action. J. Exp. Bot. 60, 1439-1463 (2009).

398 3. Kim, T.-H., Böhmer, M., Hu, H., Nishimura, N. \& Schroeder, J. I. Guard cell signal transduction network: 399 advances in understanding abscisic acid, $\mathrm{CO}_{2}$, and $\mathrm{Ca}^{2+}$ signaling. Annu. Rev. Plant Biol. 61, 561-591 (2010). 
4014 4. Murata, Y., Mori, I. C. \& Munemasa, S. Diverse stomatal signaling and the signal integration mechanism. Annu. Rev. Plant Biol. 66, 369-392 (2015).

403 5. Kollist, H., Nuhkat, M. \& Roelfsema, M. R. G. Closing gaps: linking elements that control stomatal 404 movement. New Phytol. 203, 44-62 (2014).

405 6. Roelfsema, M. R. G., Hedrich, R. \& Geiger, D. Anion channels: master switches of stress responses. Trends Plant Sci. 17, 221-229 (2012).

7. Pantoja, O. Recent advances in the physiology of ion channels in plants. Annu. Rev. Plant Biol. 72, 463495 (2021).

$409 \quad$ 8. Hedrich, R. Ion channels in plants. Physiol. Rev. 92, 1777-1811 (2012).

$4109 . \quad$ Keller, B. U., Hedrich, R. \& Raschke, K. Voltage-dependent anion channels in the plasma membrane of 411 guard cells. Nature 341, 450-453 (1989).

412 10. Schroeder, J. I. \& Keller, B. U. Two types of anion channel currents in guard cells with distinct voltage regulation. Proc. Natl. Acad. Sci. USA 89, 5025-5029 (1992).

414 11. Schroeder, J. I. et al. Cytosolic calcium regulates ion channels in the plasma membrane of Vicia faba 415 guard cells. Nature 338, 427-430 (1989).

416 12. Hedrich, R., Busch, H. \& Raschke, K. $\mathrm{Ca}^{2+}$ and nucleotide dependent regulation of voltage dependent anion channels in the plasma membrane of guard cells. EMBO J. 9, 3889-3892 (1990).

418 13. Vahisalu, T. et al. SLAC1 is required for plant guard cell S-type anion channel function in stomatal signalling. Nature 452, 487-491 (2008).

420 14. Negi, J. et al. $\mathrm{CO}_{2}$ regulator SLAC1 and its homologues are essential for anion homeostasis in plant cells. Nature 452, 483-486 (2008).

422 15. Saji, S. et al. Disruption of a gene encoding C4-dicarboxylate transporter-like protein increases ozone sensitivity through deregulation of the stomatal response in Arabidopsis thaliana. Plant Cell Physiol. 49, $2-10$ (2008).

16. Geiger, D. et al. Activity of guard cell anion channel SLAC1 is controlled by drought-stress signaling kinase-phosphatase pair. Proc. Natl. Acad. Sci. USA 106, 21425-21430 (2009). 
430 18. Brandt, B. et al. Reconstitution of abscisic acid activation of SLAC1 anion channel by CPK6 and OST1

(2012).

433 19. Maierhofer, T. et al. Site-and kinase-specific phosphorylation-mediated activation of SLAC1, a guard cell anion channel stimulated by abscisic acid. Sci. Signal. 7, 1-12 (2014).

20. Deng, Y. N. et al. Structure and activity of SLAC1 channels for stomatal signaling in leaves. Proc. Natl. Acad. Sci. USA 118, 1-8 (2021).

21. Chen, Y.-H. et al. Homologue structure of the SLAC1 anion channel for closing stomata in leaves. Nature 467, 1074-1080 (2010).

22. Meyer, S. et al. AtALMT12 represents an R-type anion channel required for stomatal movement in Arabidopsis guard cells. Plant J. 63, 1054-1062 (2010).

441 23. Sasaki, T. et al. Closing plant stomata requires a homolog of an aluminum-activated malate transporter. Plant Cell Physiol. 51, 354-365 (2010).

443 24. Jalakas, P. et al. Combined action of guard cell plasma membrane rapid- and slow-type anion channels in stomatal regulation. Plant Physiol. (2021) doi:10.1093/plphys/kiab202.

25. Gutermuth, T. et al. Tip-localized $\mathrm{Ca}^{2+}$-permeable channels control pollen tube growth via kinasedependent R- and S-type anion channel regulation. New Phytol. 218, 1089-1105 (2018).

449 27. Mumm, P. et al. C-terminus-mediated voltage gating of arabidopsis guard cell anion channel QUAC1. Mol. Plant 6, 1550-1563 (2013).

451 28. Hedrich, R. et al. Malate-sensitive anion channels enable guard cells to sense changes in the ambient $\mathrm{CO}_{2}$ concentration. Plant J. 6, 741-748 (1994).

$45329 . \quad$ Raschke, K. Alternation of the slow with the quick anion conductance in whole guard cells effected by 454 external malate. Planta 217, 651-657 (2003).

455 30. Imes, D. et al. Open stomata 1 (OST1) kinase controls R-type anion channel QUAC1 in Arabidopsis 456 guard cells. Plant J. 74, 372-382 (2013). 
457 31. Luu, K., Rajagopalan, N., Ching, J. C. H., Loewen, M. C. \& Loewen, M. E. the malate-activated

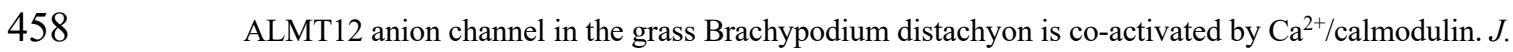
Biol. Chem. 294, 6142-6156 (2019).

460 32. Meyer, S. et al. Malate transport by the vacuolar AtALMT6 channel in guard cells is subject to multiple regulation. Plant J. 67, 247-257 (2011).

462 33. De Angeli, A., Zhang, J., Meyer, S. \& Martinoia, E. AtALMT9 is a malate-activated vacuolar chloride channel required for stomatal opening in Arabidopsis. Nat. Commun. 4, 1-10 (2013).

464 34. Kovermann, P. et al. The Arabidopsis vacuolar malate channel is a member of the ALMT family. Plant $J$. 52, 1169-1180 (2007).

466 35. Sasaki, T. et al. A wheat gene encoding an aluminum-activated malate transporter. Plant J. 37, 645-653 (2004).

468 36. Hoekenga, O. A. et al. AtALMT1, which encodes a malate transporter, is identified as one of several genes critical for aluminum tolerance in Arabidopsis. Proc. Natl. Acad. Sci. USA 103, 9738-9743 (2006). controlling aluminum tolerance at the Alt4 Locus of Rye (Secale cereale L.). Genetics179, 669-682 genes from rape encode aluminum-activated malate transporters that enhance the aluminum resistance of plant cells. Plant Physiol. 142, 1294-1303 (2006).

476 39. Piñeros, M. A. et al. Not all ALMT1-type transporters mediate aluminum-activated organic acid responses: The case of ZmALMT1 - An anion-selective transporter. Plant J. 53, 352-367 (2008).

478 40. Ligaba, A., Maron, L., Shaff, J., Kochian, L. \& Piñeros, M. Maize ZmALMT2 is a root anion transporter that mediates constitutive root malate efflux. Plant, Cell Environ. 35, 1185-1200 (2012).

480 41. Bai, Y. et al. A natural mutation-led truncation in one of the two aluminum-activated malate transporter481 like genes at the Ma locus is associated with low fruit acidity in apple. Mol. Genet. Genomics 287, 663678. (2012).

483 42. De Angeli, A. et al. The vacuolar channel VvALMT9 mediates malate and tartrate accumulation in berries 
485 43. Rudrappa, T., Czymmek, K. J., Paré, P. W. \& Bais, H. P. Root-secreted malic acid recruits beneficial soil $486 \quad$ bacteria. Plant Physiol. 148, 1547-1556 (2008).

487 44. Takanashi, K. et al. A dicarboxylate transporter, LjALMT4, mainly expressed in nodules of lotus $488 \quad$ japonicus. Mol. Plant-Microbe Interact. 29, 584-592 (2016).

489 45. Xu, M. et al. The barley anion channel, HvALMT1, has multiple roles in guard cell physiology and grain 490 metabolism. Physiol. Plant. 153, 183-193 (2015).

491 46. Heng, Y. et al. OsALMT7 maintains panicle size and grain yield in rice by mediating malate transport. $492 \quad$ Plant Cell 30, 889-906 (2018).

493 47. Holm, L. DALI and the persistence of protein shape. Protein Sci. 29, 128-140 (2020).

494 48. Furuichi, T. et al. An extracellular hydrophilic carboxy-terminal domain regulates the activity of 495 TaALMT1, the aluminium-activated malate transport protein of wheat. Plant J. 64, 47-55 (2010).

496 49. Smart, O. S., Neduvelil, J. G., Wang, X., Wallace, B. A. \& Sansom, M. S. P. HOLE: A program for the 497 analysis of the pore dimensions of ion channel structural models. J. Mol. Graph. 14, 354-360 (1996).

498 50. Peng, W. et al. Characterization of the soybean GmALMT family genes and the function of GmALMT5 499 in response to phosphate starvation. J. Integr. Plant Biol. 60, 216-231 (2018).

500 51. Jumper, J. et al. Highly accurate protein structure prediction with AlphaFold. Nature (2021) doi:10.1038/s41586-021-03819-2.

52. Altschul, S. Gapped BLAST and PSI-BLAST: a new generation of protein database search programs. Nucleic Acids Res. 25, 3389-3402 (1997).

504 53. Wang, X.-H. et al. Structural basis for the activity of TRIC counter-ion channels in calcium release. Proc. Natl. Acad. Sci. USA 116, 4238-4243 (2019).

506 54. Mastronarde, D. N. Automated electron microscope tomography using robust prediction of specimen 507 movements. J. Struct. Biol. 152, 36-51. (2005).

508 55. Wu, C., Huang, X., Cheng, J., Zhu, D. \& Zhang, X. High-quality, high-throughput cryo-electron 509 microscopy data collection via beam tilt and astigmatism-free beam-image shift. J. Struct. Biol. 208, $510107396(2019)$.

511 56. Zivanov, J. et al. New tools for automated high-resolution cryo-EM structure determination in RELION- 
513 57. Punjani, A., Rubinstein, J. L., Fleet, D. J. \& Brubaker, M. A. cryoSPARC: algorithms for rapid

514 unsupervised cryo-EM structure determination. Nat. Methods 14, 290-296 (2017).

$515 \quad 58 . \quad$ Asarnow, D., Palovcak, E. \& Cheng, Y. UCSF pyem. (2021).

516 59. Pettersen, E. F. et al. UCSF Chimera--a visualization system for exploratory research and analysis. $J$.

517 Comput. Chem. 25, 1605-1612 (2004).

518 60. Zheng, S. Q. et al. MotionCor2: anisotropic correction of beam-induced motion for improved cryo-

519 electron microscopy. Nat. Methods 14, 331-332 (2017).

520 61. Zhang, K. Gctf: Real-time CTF determination and correction. J. Struct. Biol. 193, 1-12 (2016).

521 62. Zhang, K., and Sun, F. Gautomatch: an efficient and convenient gpu-based automatic particle selection

522 program. https://www2.mrc-lmb.cam.ac.uk/download/gautomatch-056. (2011).

523 63. Liebschner, D. et al. Macromolecular structure determination using X-rays, neutrons and electrons: recent 524 developments in it Phenix. Acta Crystallogr. Sect. D 75, 861-877 (2019).

525 64. Kucukelbir, A., Sigworth, F. J. \& Tagare, H. D. Quantifying the local resolution of cryo-EM density 526 maps. Nat. Methods 11, 63-65 (2014).

527 65. Emsley, P., Lohkamp, B., Scott, W. G. \& Cowtan, K. Features and development of Coot. Acta 528 Crystallogr. Sect. D. 66, 486-501 (2010).

529 66. Gabler, F. et al. Protein sequence analysis using the MPI bioinformatics toolkit. Curr. Protoc.

$530 \quad$ Bioinforma. 72, e108 (2020).

531 67. Barad, B. A. et al. EMRinger: Side chain-directed model and map validation for 3D cryo-electron 532 microscopy. Nat. Methods 12, 943-946 (2015).

533 68. Pettersen, E. F. et al. UCSF ChimeraX: Structure visualization for researchers, educators, and developers.

534 Protein Sci. A Publ. Protein Soc. 30, 70-82 (2021).

535 69. Bi, G. et al. The ZAR1 resistosome is a calcium-permeable channel triggering plant immune signaling. Cell 184, 3358-3360 (2021). 


\section{METHODS}

\section{Bioinformatics analysis of ALMT proteins}

540 Sequences related to QUAC1/ALMTs were searched and analyzed by using PSI-BLAST ${ }^{52}$.

541 Searches at $\mathrm{E}<5 * 10^{-3}$ starting with thirteen individual Arabidopsis ALMTs identified a 542 common pool of over 1,700 plant ALMT-related protein sequences, which were pooled 543 together and used for sub-classification into families and subfamilies. Detailed information is 544 reported in the footnotes to the Extended Data Table 1.

\section{Cloning and expression of QUAC1/ALMT12 in yeast $S$. pombe}

547 Full-length coding sequences of six plant QUAC1s from rice, wheat, corn, soybean, tomato and 548 cotton, were cloned into a modified pREP1 vector with a C-terminal $10 \times$ His tag. The resulting 549 constructs were transformed into a leucine auxotrophic $S$. pombe strain, and the transformants 550 were selected on the standard EMM plates without leucine, as previously described ${ }^{53}$. To 551 prevent protein expression during strain growth, $25 \mu \mathrm{M}$ thiamine was added to inhibit the 552 promoter.

\section{Scaled-up production and purification of $G m Q U A C 1$}

555 To prepare the seed, transformed cells were inoculated into $100 \mathrm{ml}$ EMM culture medium 556 supplemented with $25 \mu \mathrm{M}$ thiamine and were shaken at $200 \mathrm{rpm}$ and $30^{\circ} \mathrm{C}$ for $24 \mathrm{hr}$. For protein 557 expression and scaled-up production, the seed cells were collected by centrifugation and 558 washed with sterile water twice before inoculation to a culture of $500 \mathrm{ml}$. After $12 \mathrm{hr}$ growth, $559500 \mathrm{ml}$ of fresh medium was supplemented, and the culture continued to grow for an additional $56024 \mathrm{hr}$. Cells were harvested through centrifugation for $20 \mathrm{~min}$ at 4,500 rpm.

561 For protein purification, cells were re-suspended in lysis buffer $(50 \mathrm{mM}$ Tris- $\mathrm{HCl} \mathrm{pH} 8.0$, $562200 \mathrm{mM} \mathrm{NaCl}, 2.5 \%$ glycerol, $1 \mu \mathrm{g} / \mathrm{ml}$ aprotinin, $1 \mu \mathrm{g} / \mathrm{ml}$ leupeptin, $1 \mu \mathrm{g} / \mathrm{ml}$ pepstatin, $2 \mathrm{mM}$ 563 PMSF and $2 \mathrm{mM} \mathrm{DTT}$ ) and lysed using a high-pressure cell disrupter (JNBIO) with 4 passes 564 at $\sim 18,000$ psi. Cell debris was removed by centrifugation at 12,000 rpm for $15 \mathrm{~min}$, and the 565 supernatant was subjected to a further ultra-centrifugation at $41,000 \mathrm{rpm}$ for $1 \mathrm{hr}$. The 
membrane was collected and homogenized in a solubilization buffer $(50 \mathrm{mM}$ Tris- $\mathrm{HCl} \mathrm{pH} 8.0$,

$567200 \mathrm{mM} \mathrm{NaCl}$ and $2.5 \%$ glycerol) and incubated with a final concentration of $1.0 \%$ DDM and

$568 \quad 0.02 \%$ CHS by gentle stirring for $1 \mathrm{hr}$ at $4^{\circ} \mathrm{C}$. After ultra-centrifugation at 35,000 rpm for 50

$569 \min$, the resulting supernatant was purified by $\mathrm{Ni}^{2+}$-affinity column pre-equilibrated with the 570 same solubilization buffer supplemented with $0.05 \%$ DDM and $2 \mathrm{mM}$ TCEP. After 20 columns

571 volume of buffer wash, the protein was eluted with $350 \mathrm{mM}$ imidazole in the solubilization

572 buffer. The resulting $G m \mathrm{QUAC1}$ protein, without removal of His-tag, was concentrated to $\sim 10$

$573 \mathrm{mg} / \mathrm{ml}$ and loaded onto a Superose6 (10/300) gel-filtration column for further purification and

574 detergent exchange. The gel-filtration buffer contained $20 \mathrm{mM}$ Tris, $150 \mathrm{mM} \mathrm{NaCl}(\mathrm{pH} 8.0$,

575 adjusted with $\mathrm{HCl}$ ) or $75 \mathrm{mM} \mathrm{L-malate}(\mathrm{pH} 8.0$, adjusted with $\mathrm{NaOH}$ ), and $0.005 \%$ LMNG.

576 The elution fractions were analyzed by SDS-PAGE, and the peak fractions were collected and 577 concentrated for functional analysis $(\sim 2 \mathrm{mg} / \mathrm{ml})$ or making cryo-EM grids $(\sim 5 \mathrm{mg} / \mathrm{ml})$.

\section{Cryo-EM grid preparation and data acquisition}

$5804 \mu \mathrm{l}$ of $\mathrm{GmQUAC1}{ }^{\text {malate }}$ proteins (purified in $75 \mathrm{mM}$ L-malate) were applied to newly glow581 discharged holy carbon film grids (Au R1.2/1.3, 300 meshes, Quantifoil, Germany). The grids 582 were blotted with force 3 and blotting time of $9.0 \mathrm{~s}$ at $100 \%$ humidity and $4{ }^{\circ} \mathrm{C}$ and were vitrified 583 by plunge freezing into liquid ethane using Vitrobot Mark IV (Thermo Fisher Scientific, USA).

584 All movies were collected on a Titan Krios G2 transmission electron microscope (Thermo 585 Fisher Scientific, USA) operated at $300 \mathrm{KV}$, equipped with a Gatan K2 Summit direct detection camera (Gatan Company, USA) and a post-column GIF energy filter.

Data collection was performed on EF-TEM mode with a silt width of $20 \mathrm{e}^{-} \cdot \mathrm{V}$. The magnification was set to a nominal $130,000 x$, corresponding to a calibrated pixel size of 1.04

$589 \AA /$ pixel at the specimen level $(0.52 \AA /$ pixel in super-resolution mode). Images were recorded 590 using SerialEM (version 3.8.4) ${ }^{54}$ with a beam-image shift method ${ }^{55}$. During the 8 s exposure, 59132 frames were collected with a dose of around $60 \mathrm{e}^{-} / \AA^{2}$. A total of 6,189 movie stacks were 592 collected, with defocus ranging from $-1.2 \mu \mathrm{m}$ to $-2.2 \mu \mathrm{m}$. 


\section{Image processing and model building}

595 For cryo-EM image processing, all main steps were performed using RELION $3.0{ }^{56}$ or 596 cryoSPARC $3 \cdot 1^{57}$. Pyem ${ }^{58}$ and UCSF Chimera ${ }^{59}$ were used for format conversion of data files 597 and reconstructions analysis, respectively.

598 In brief, 6,189 movie stacks were aligned by $5 \times 5$ patches with dose weighting and binned 599 to $1.04 \AA$ using Motioncor $2{ }^{60}$. The contrast transfer function parameters were estimated using $600 \mathrm{GCTF}^{61}$. Micrographs with defects in the thon rings were discarded. 2,987,283 candidate 601 particles were initially picked using Gautomatch without template ${ }^{62}$, and followed by several 602 rounds of 2D classification in cryoSPARC, giving a set of 2,136,754 high-quality particles. A 603 rough initial model was generated by a subset of 100,000 particles. Subsequentially, six rounds 604 of 3D classification were performed at C1 symmetry. Three parallel 3D classifications were 605 performed within each round, and the resulting good particles were combined for sequential 606 round classification. After that, a set of 428,433 particles that generated a map of $4.0 \AA$ using 607 NU-Refinement in cryoSPARC were subjected to Bayesian polishing. Three rounds of 3D 608 classification were further performed as described below. Each round contains an NU609 Refinement at C2 symmetry in cryoSPARC followed by an alignment-free 3D classification 610 with a micelle-free mask in Relion. Finally, NU-refinement of 169,576 particles yielded a 611 reconstruction with a resolution of $3.5 \AA$, based on the gold-standard Fourier Shell Correlation 612 (FSC) using the 0.143 criterion. Density modification was performed using PHENIX ${ }^{63}$. Local 613 resolution was determined using ResMap ${ }^{64}$.

614 A de novo atomic model was built manually in Coot ${ }^{65}$ using the predicted secondary 615 structure from MPI quick2d ${ }^{66}$. The model was further refined using PHENIX ${ }^{63}$. The final 616 model was validated using EMRinger ${ }^{67}$. All structure figures were prepared in UCSF 617 ChimeraX ${ }^{68}$.

\section{The two-electrode voltage-clamp recordings}

620 The TEVC recordings were conducted as previously described ${ }^{20}$. In brief, all cDNAs for $621 G m$ QUAC1, including wild-type or mutants, were cloned into plasmid pGHME2 for expression 
622 in Xenopus laevis oocyte. Linearised plasmids were used to generate cRNAs using T7

623 polymerase. 36 nanograms of cRNA of each construct were injected into isolated oocytes.

624 Oocytes were then incubated at $18^{\circ} \mathrm{C}$ for $\sim 48 \mathrm{hr}$ in ND96 buffer $\left(96 \mathrm{mM} \mathrm{NaCl}, 1.8 \mathrm{mM} \mathrm{CaCl}_{2}\right.$,

$6251 \mathrm{mM} \mathrm{MgCl}_{2}, 2 \mathrm{mM} \mathrm{KCl}, 5 \mathrm{mM}$ HEPES-Na, $\mathrm{pH}$ 7.5).

626 Using a Oocyte Clamp OC-725C amplifier (Warner Instruments) and a Digidata 1550 B

627 low-noise data acquisition system with pClamp software (Molecular Devices), TEVC

628 recordings were performed in the bath solutions: $10 \mathrm{mM} \mathrm{MES/Tris} \mathrm{pH}$ 5.6, $1 \mathrm{mM}$

$629 \mathrm{Ca}$ (gluconate) $)_{2}, 1 \mathrm{mM} \mathrm{Mg}$ (gluconate) $)_{2}, 70 \mathrm{mM} \mathrm{Na(gluconate),} 1 \mathrm{mM} \mathrm{LaCl}_{3}, 30 \mathrm{mM} \mathrm{NaCl}^{\text {or }}$

$630 \mathrm{NaNO}_{3}$, or $30 \mathrm{mM} \mathrm{L}$-malate (pH 5.6, prepared from L-malic acid with $\mathrm{NaOH}$ ). Osmolarity was

631 adjusted to $\sim 220 \mathrm{mOsmol} * \mathrm{~kg}^{-1}$ with D-Sorbitol. The microelectrode solutions contained $3 \mathrm{M}$

$632 \mathrm{KCl}$, and the bath electrode was a $3 \mathrm{M} \mathrm{KCl}$ agar bridge. Voltage-clamp currents were measured

633 in response to $200 \mathrm{~ms}-$ long voltage steps to test potentials that ranged from $+60 \mathrm{mV}$ to -180

$634 \mathrm{mV}$ in $20 \mathrm{mV}$ decrement. Prior to each voltage step, the membrane was held at $+60 \mathrm{mV}$ for 50

$635 \mathrm{~ms}$, and following each voltage step, the membrane was returned to $-180 \mathrm{mV}$ for $50 \mathrm{~ms}$. I-V

636 relations for $G m$ QUAC1 channels were generated from currents measured at $5 \mathrm{~ms}$ after the

637 beginning (Instantaneous currents, $\mathrm{I}_{\text {inst }}$ ) or before the end (Steady-state currents, $\mathrm{I}_{\mathrm{ss}}$ ) of each test

638 voltage step. Three independent batches of oocytes were investigated and showed consistent

639 findings. Data from one representative oocyte batch were shown. The recordings were analyzed

640 using Clampfit 10.6 (Molecular Devices) and Prism (ver. 5.0, GraphPad).

641

642

643

644

645

646

647

648

649

\section{The planar lipid bilayer recordings}

Lipid bilayer experiments were conducted as previously described in our study of the ZAR1 channel ${ }^{69}$. The purified $\mathrm{GmQUAC1}$ (in $150 \mathrm{mM} \mathrm{NaCl}$ or $75 \mathrm{mM}$ L-malate), at a concentration of $1.5 \sim 2.5 \mu \mathrm{g} / \mathrm{ml}$, was fused into planar lipid bilayers formed by painting a lipid mixture of phosphatidylethanolamine (DOPE) and phosphatidylcholine (DOPC) (Avanti Polar Lipids) in a 5:3 ratio in decane across a $200 \mu \mathrm{m}$ hole in a polystyrene partition separating the internal and external solutions in polysulfonate cups (Warner Instruments). The trans chamber (1.0 ml), representing the extracellular compartment, was connected to the head stage input of a bilayer 
650 voltage-clamp amplifier. The cis chamber $(1.0 \mathrm{ml})$, representing the cytoplasmic compartment,

651 was held at virtual ground. Solutions used for I-V relationship analysis were as follows: 150

$652 \mathrm{mM} \mathrm{NaCl}$ or $150 \mathrm{mM} \mathrm{NaNO}_{3}$. All solutions were buffered with $20 \mathrm{mM}$ Tris (pH 8.0). Purified

653 proteins were added to the cis side and fused with the lipid bilayer.

654 Currents were recorded every 1 2 min after application of the voltage to the trans side.

655 Single-channel currents were recorded using a Bilayer Clamp BC-525D (Warner Instruments),

656 filtered at $800 \mathrm{~Hz}$ using a Low-Pass Bessel Filter 8 Pole (Warner Instruments) and digitized at

$6574 \mathrm{kHz}$. All experiments were performed at room temperature $\left(23 \pm 2^{\circ} \mathrm{C}\right)$. The recordings were

658 analyzed using Clampfit 10.6 (Molecular Devices) and Prism (ver. 5.0, GraphPad).

\section{Biochemical characterization of CHD of GmQUAC1}

661 cDNAs encoding the C-terminal cytoplasmic helical domain of GmQUAC1 (wild-type or 662 mutants) were subcloned into a modified bacterial expression vector pET-24a, with a C663 terminal $10 \times$ His tag. Transformed E. coli BL21 (DE3) cells were grown in a TB medium 664 containing Kanamycin $(50 \mu \mathrm{g} / \mathrm{ml})$. Protein expression was induced in cells grown to an optical 665 density at OD600 of $\sim 0.8$ with $0.4 \mathrm{mM}$ isopropyl $\beta$-D-thiogalactoside (IPTG) and followed by 666 overnight cell growth at $16^{\circ} \mathrm{C}$. Cells were collected and subsequently lysed using a high667 pressure cell disrupter (JNBIO) with 3 passes at $\sim 15,000$ psi. The target protein was purified 668 by using $\mathrm{Ni}^{2+}$ affinity chromatography and further analyzed by Superdex75 (10/300) gel 669 filtration chromatography and SDS-PAGE.

\section{The three-dimensional structure modelling using AlphaFold2}

Briefly, the sequence for AlphaFold prediction was generated by two individual

$673 G m$ QUAC1 sequences, connecting by a linker of tandemly repeated sequence of (GSGS $)_{50}$.

674 Running with the local installed AlphaFold2 program and the CASP14 preset and databases (as 675 of July 23, 2021), five dimer models were generated, with their pLDDT (predicted 1DDT-C $\alpha$ ) 676 scores ranging from 64.6 to 68.8. All the AlphaFold predicted structures (AF-structure) are nearly identical, with apparent two-fold symmetry. The two protomers in the top-rank dimer 
678 model (scored 68.8) display an r.m.s.d of $0.92 \AA$, was selected for further structural comparison

679 with the cryo-EM structure. For a better view, the disordered (residues 1-35, 394-454 and 507-

680 537) regions were removed in the final model.

681

682 QUANTIFICATION AND STATISTICAL ANALYSIS

683 In all figure legends, $\mathrm{n}$ represents the number of independent biological replicates. All

684 quantitative data were presented as mean $\pm \mathrm{SEM}$.

685

686

DATA AND CODE AVAILABILITY

687 All data are available in the manuscript or the supplementary material. The accession number

688 for the 3D cryo-EM density map reported in this paper is XXX, and the Protein Data Bank

689 (PDB) accession code for the coordinate is XXX.

690

691 ADDITIONAL RESOURCES

692 This study did not generate any additional resources.

693 
a

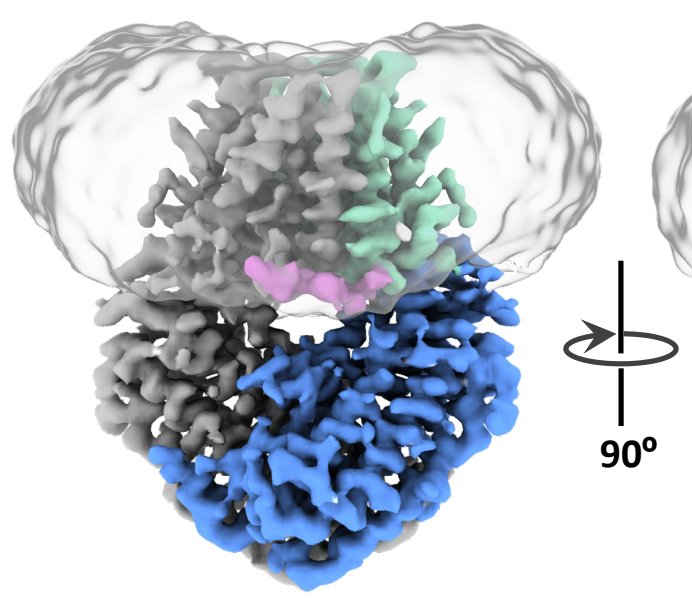

$153 \quad 218$

pre-TM TMD

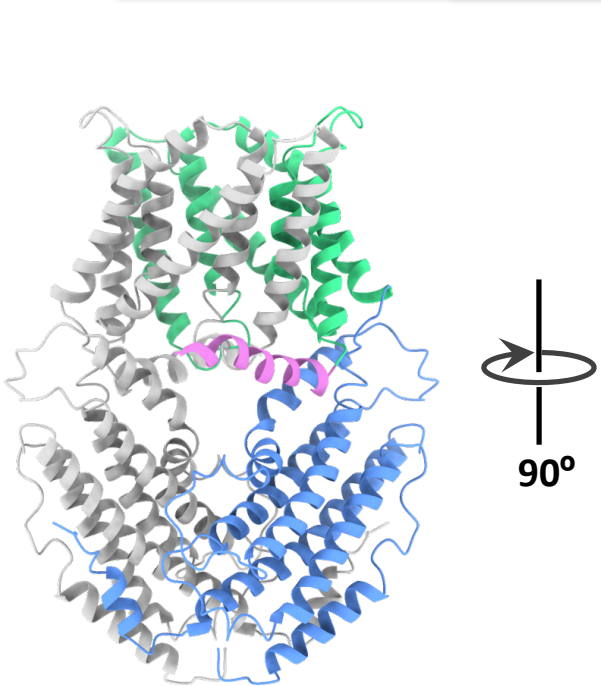

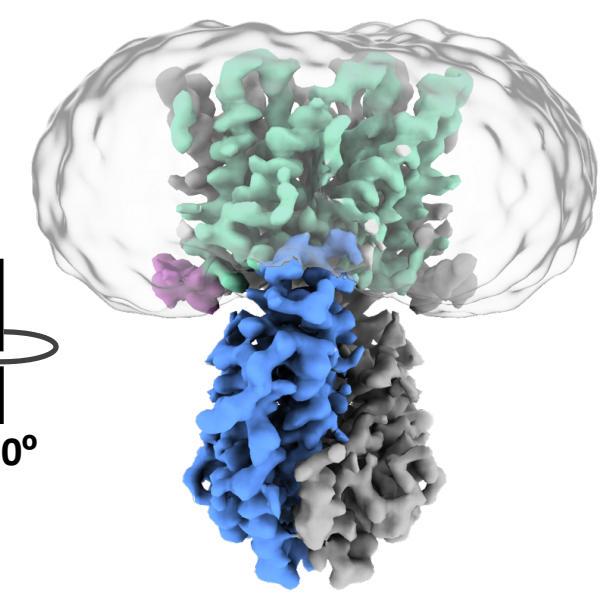

iv

CHD

$\mathrm{CH}$

C

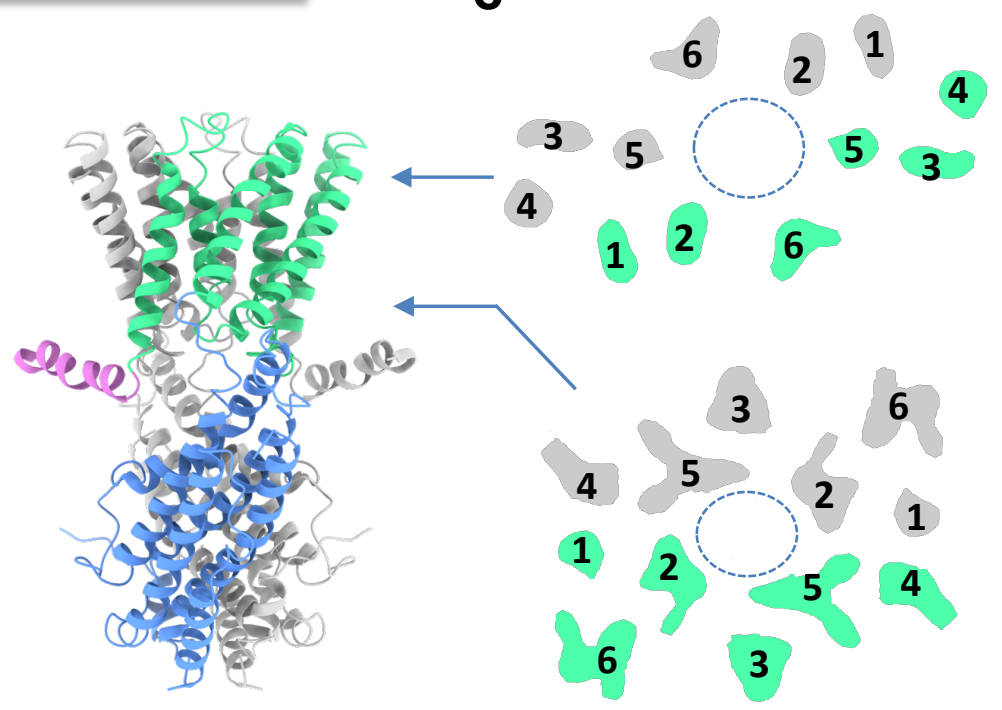

b

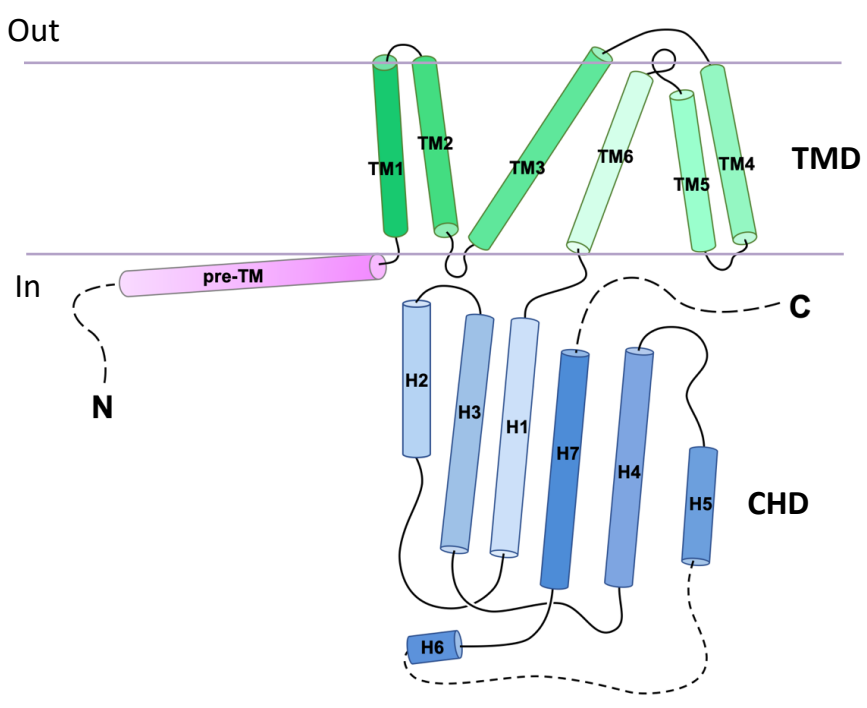

d

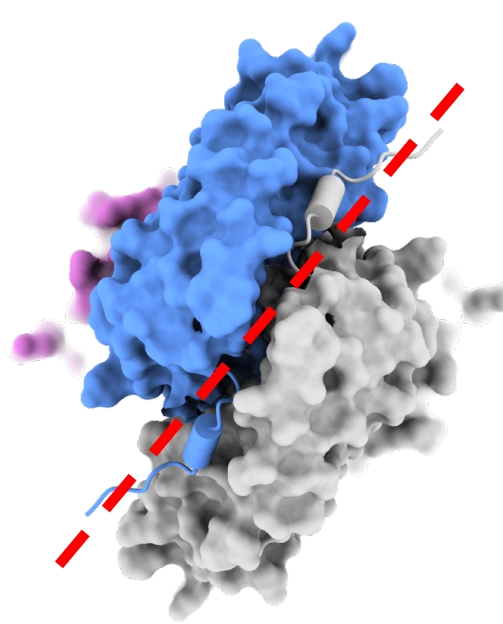

Fig. 1 Architechture of the QUAC1 channel

a, Overall structure of the GmQUAC1 channel. The cryo-EM density maps (top) and ribbons drawings (bottom) are shown, with the preTM helix in pink, the transmembrane domain (TMD) in green and the cytoplasmic helical domain (CHD) in blue in one promoter. Another promoter is coloured in grey.

b, Topology of the GmQUAC1 protomer. The six helices in TMD are marked by TM1-6, and the seven helices in CHD are marked as H1-

7. The membrane boundary is shown as grey lines. The disordered regions are indicated as dashed lines.

c, Top view of the cross-section of the transmembrane layer at the indicated positions by the arrow lines in (a). The elliptical dashed line marks the pore, and the TMs are indicated.

d, Bottom view of the C-terminal dimeric domains, colored as in (a). The domain-swapped finger helix H6 is shown as cylinder cartoon, and a red dash line marks the dimeric interface of the CHD. 

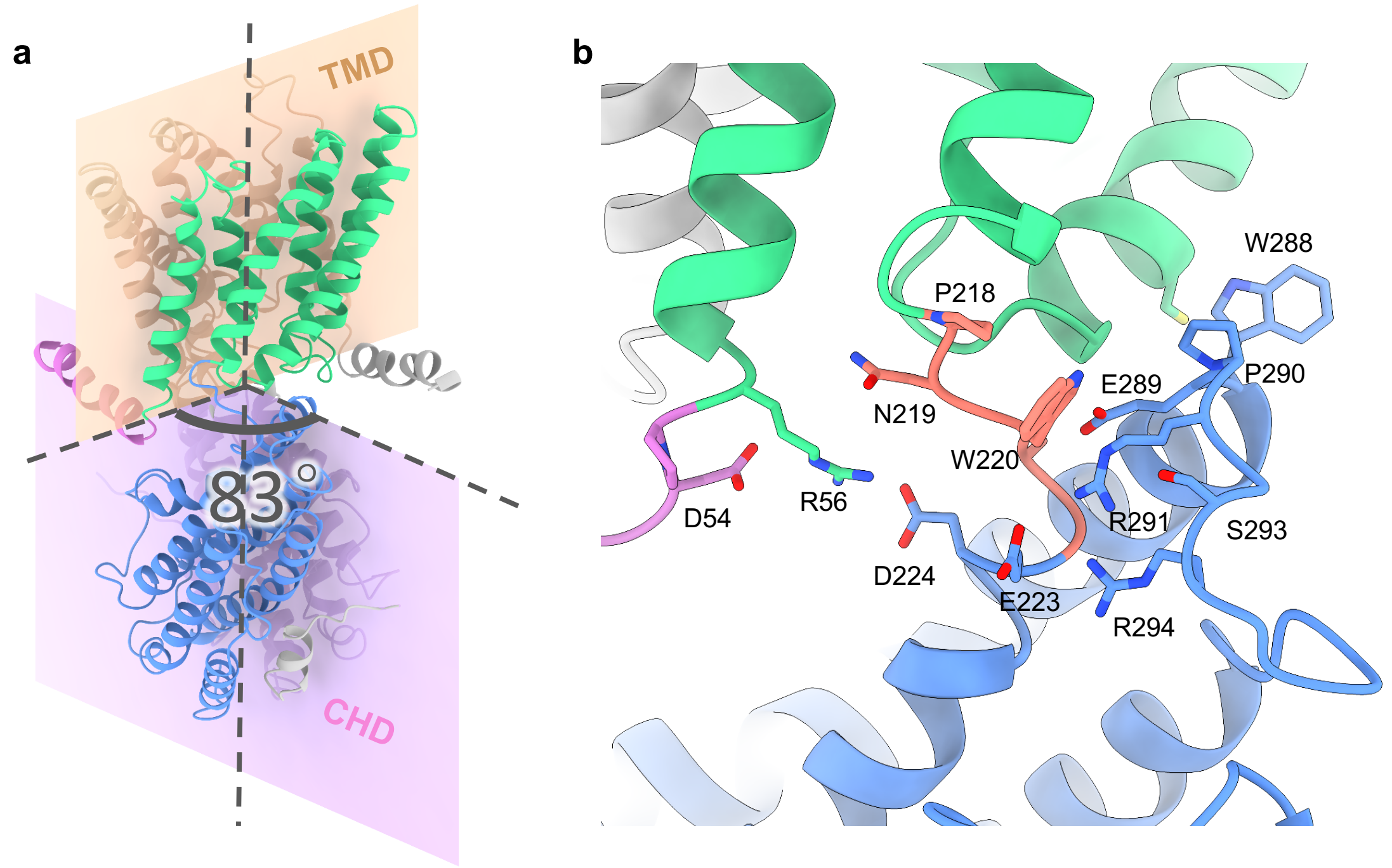

Fig. 2 The twisted bi-layer architecture of the QUAC1 channel

a, The dimeric interfaces of the TMD and CHD of the GmQUAC1 channel. These dimeric interfaces are nearly perpendicular to each other, forming a dihedral angle of $\sim 83^{\circ}$. The ribbon model is colored as in Fig. 1a.

b, The layer interface between the TMD and CHD. The TMD portion connects to the CHD portion via a highly conserved PXWXG motif (P218-N219-W220-S221-G222, in salmon), which is interact with another characteristic motif WEP (W288-E289-P290, located between helix H2 and H3, in blue). Other surrounding charged residues at the interface are also shown in sticks, including R56, R291, R294, D54, E223, D224 and E289. The ribbon model is colored as in (a). 
a

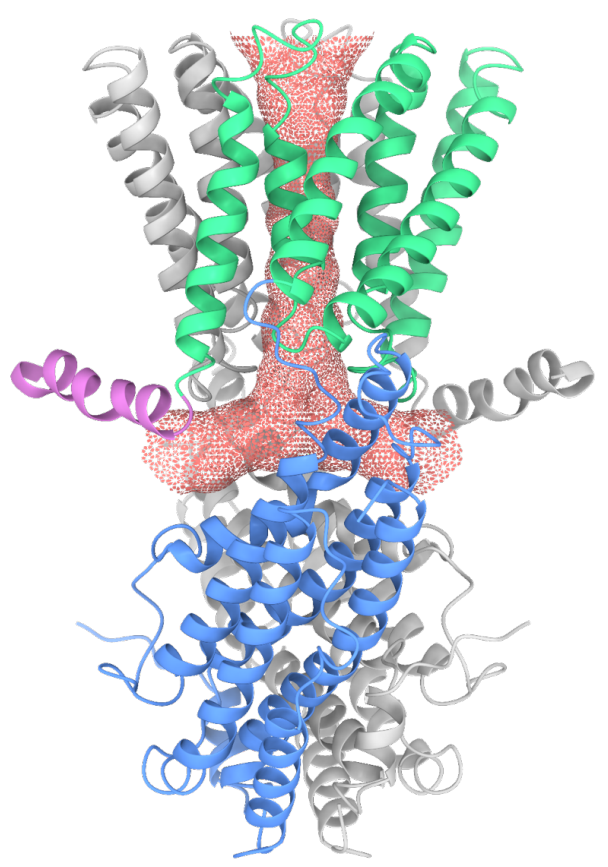

C

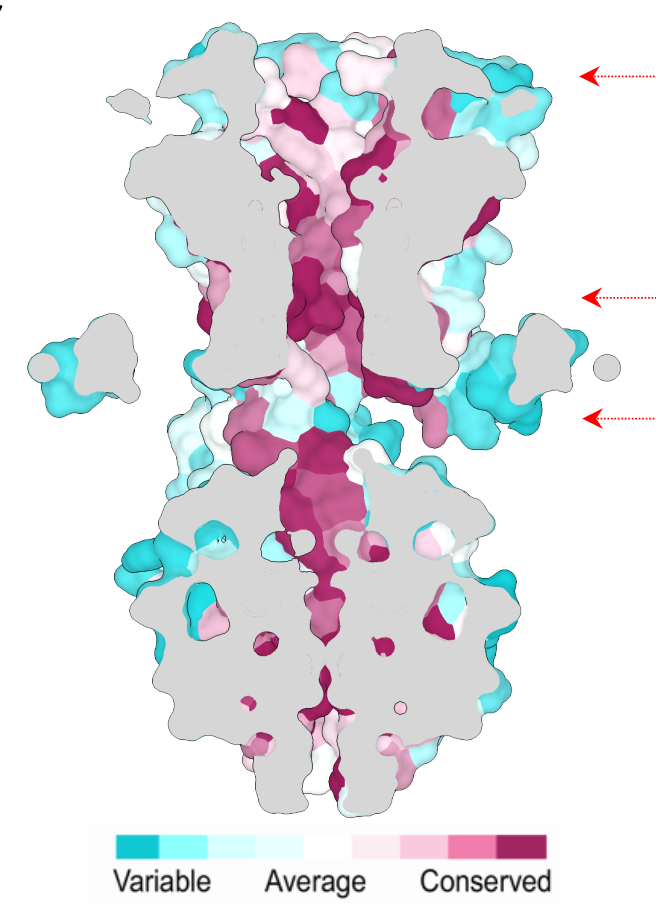

b

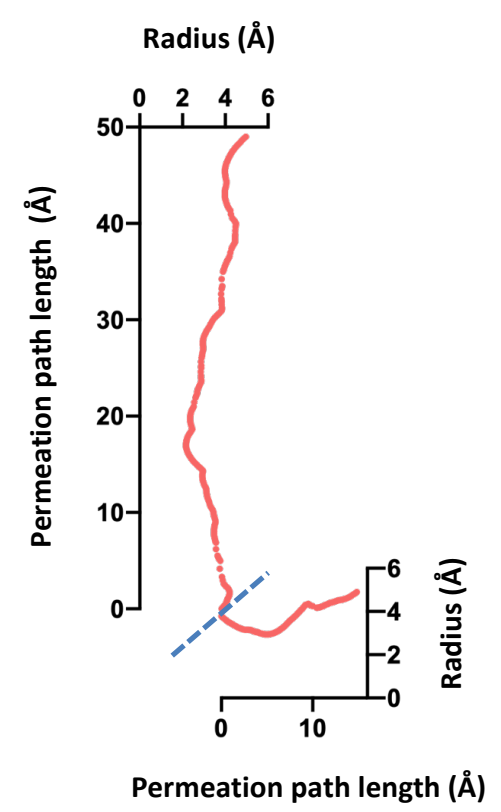

e
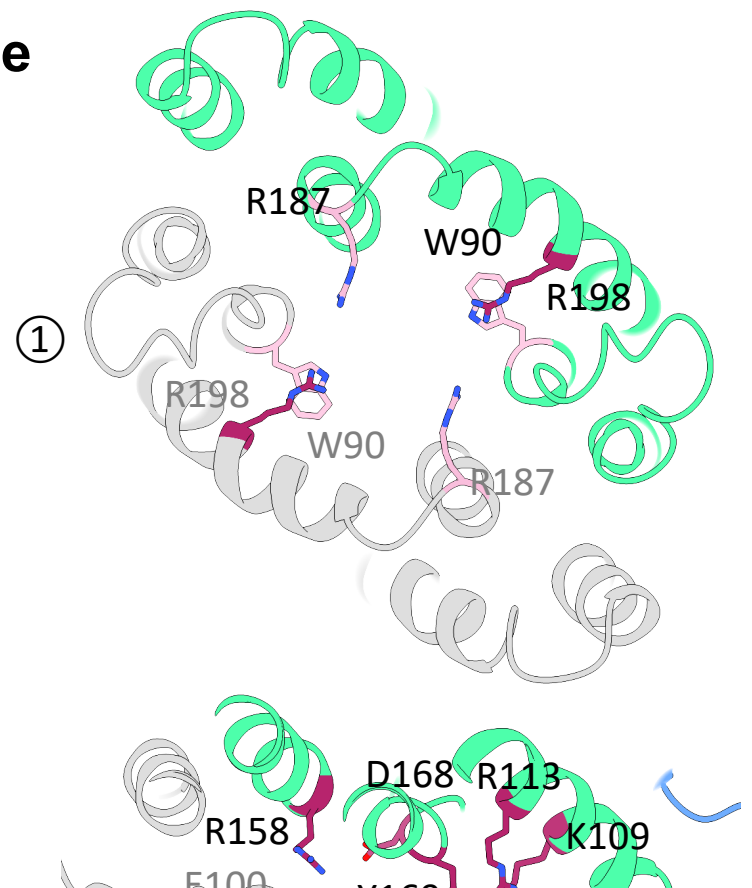

(2)

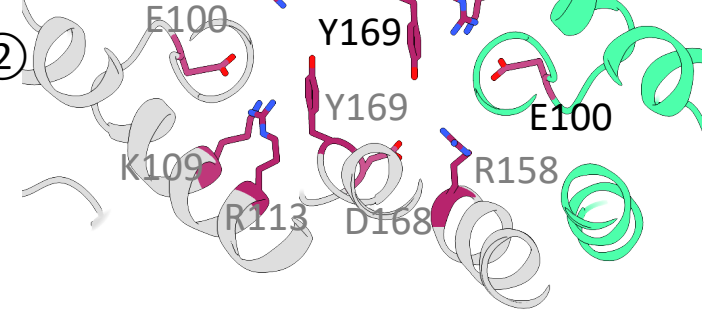

(2)

d
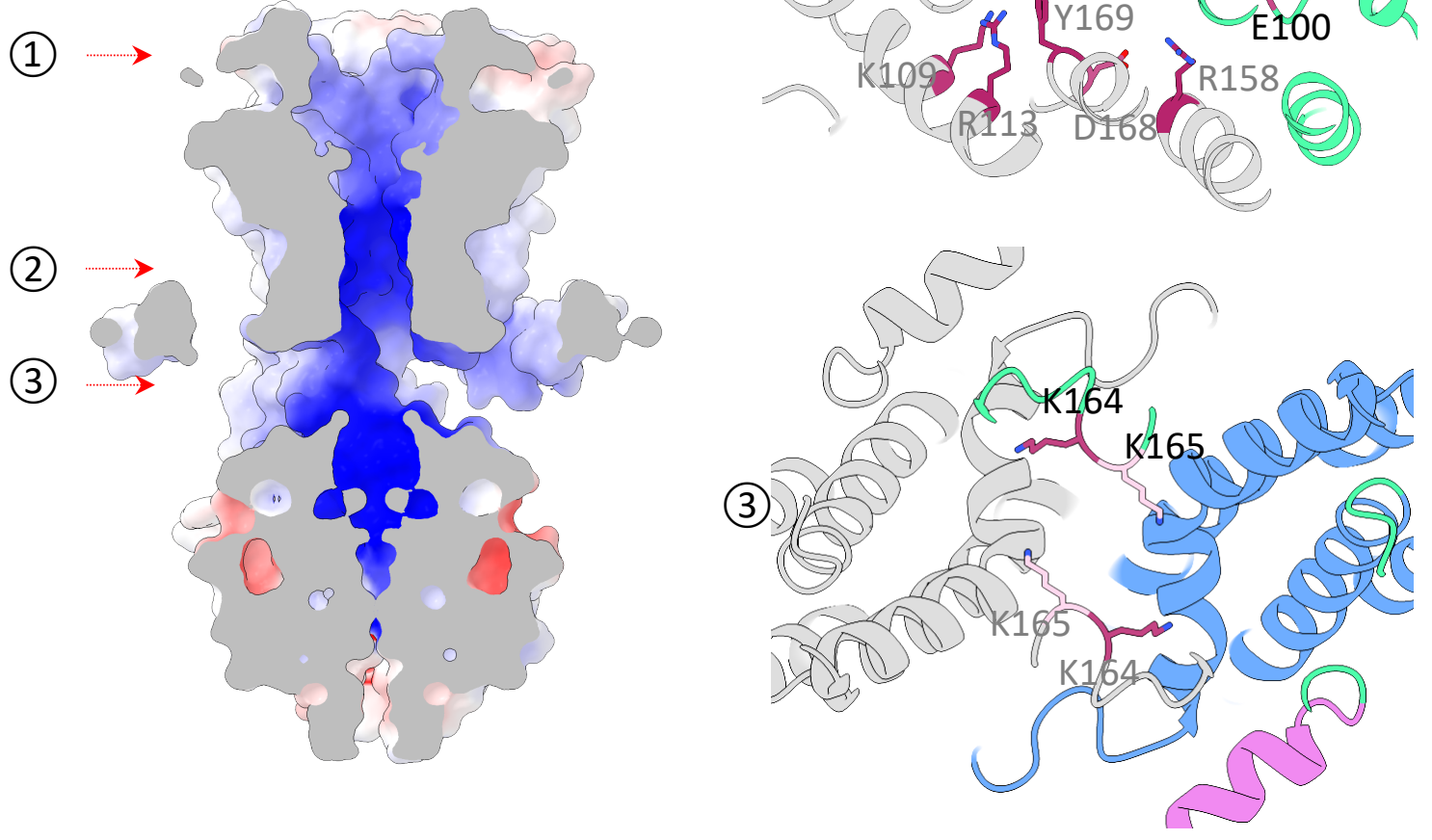

\section{Fig. 3 Structural feature of the QUAC1 channel pore}

a, The pore-lining surface was computed by the program HOLE and drawn into a ribbon model of the GmQUAC1 pore. We used a simple van der Waals surface for the protein and the program default probe radius of $1.15 \AA$. The T-shaped pore is shown in salmon dots.

b, Plot of the pore radius as a function of the pore axis.

$\mathbf{c}$ and $\mathbf{d}$, Side views of the cross-section through the ion-conducting pore. The molecular surface colored with sequence conservation for 137 non-redundant proteins of the QF2B subfamily is shown in (c) and the molecular surface colored with electrostatic potential is shown in (d). e, Top views of the cross-section through the ion-conducting pore at three indicated positions by the arrow in (c) and (d). The ribbon is colored as in (a). The conserved pore-lining charged residues are shown in sticks, coloured with the sequence conservation as in (c). 

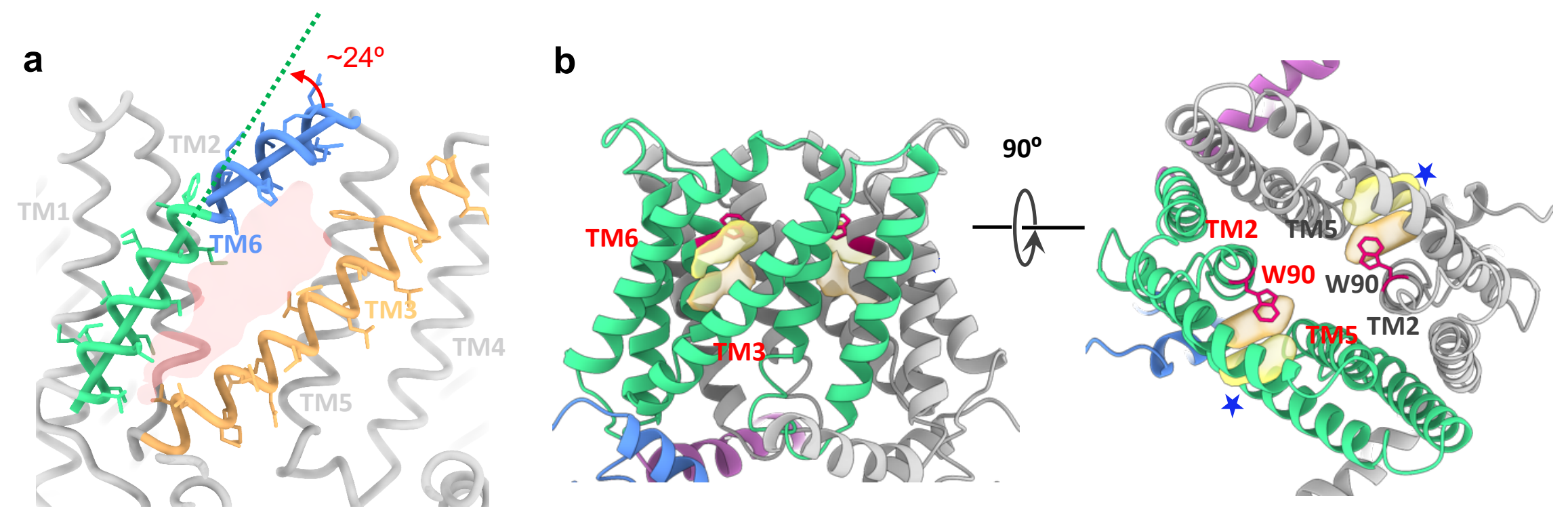

Fig. 4 The lateral fenestration in the QUAC1 channel

a, The lateral fenestration, with a dimension of $\sim 6 \AA \times 20 \AA$, is shown in transparent red, which is formed by TM2, TM3, TM5 and TM6 from one protomer. A kink $\left(\sim 24^{\circ}\right)$ in TM6 is indicated.

b, The unidentified densities (in yellow) within the fenestration (indicated by arrows). The side view (right) and top view (left) are shown. The W90 residues are shown as red sticks. The locations of fenestration are indicated with a bule stars in the top view. 
a

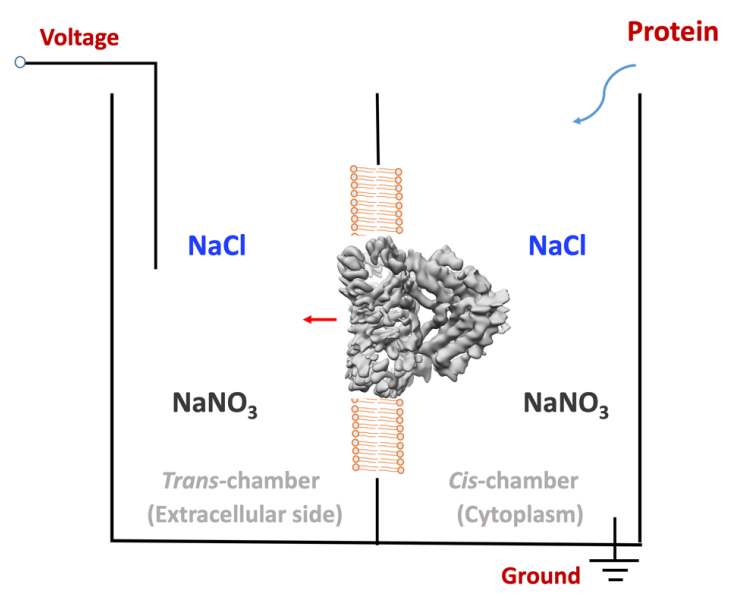

C

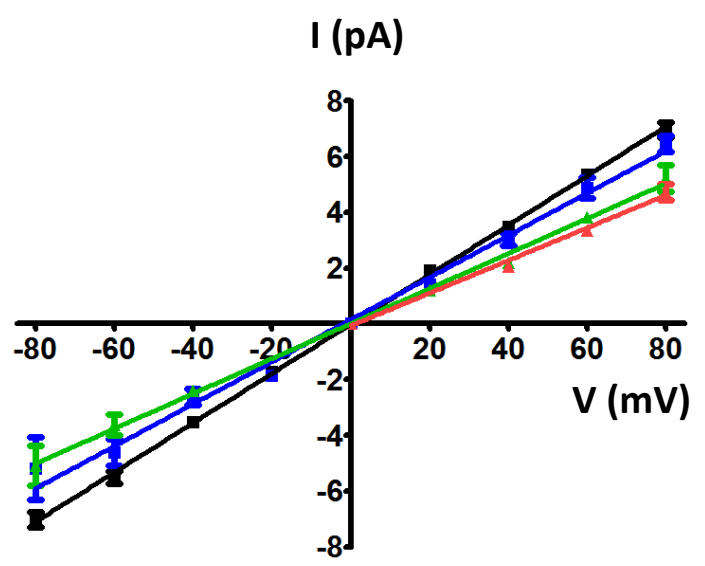

b
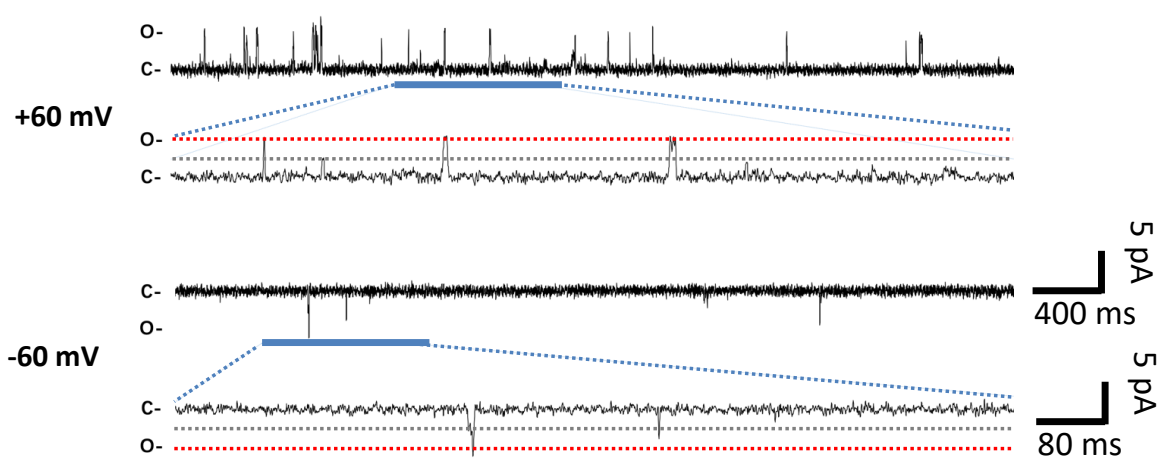

d

Po

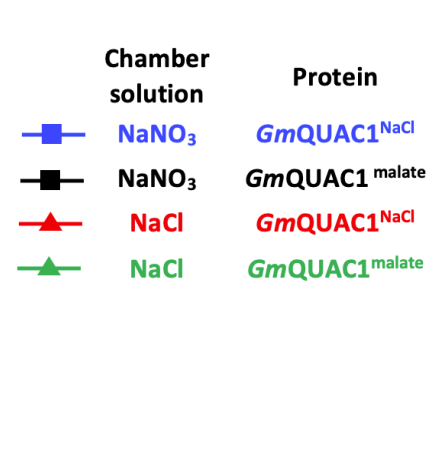

\section{Fig. 5 Single channel analysis of the QUAC1 channel in planar lipid bilayer}

a, The schematic illustration of single-channel recording of GmQUAC1 channel in a planar lipid bilayer. The chambers were filled with $1.0 \mathrm{ml}$ of symmetrical solutions of $150 \mathrm{mM} \mathrm{NaCl}$ or $150 \mathrm{mM} \mathrm{NaNO}_{3}$, and the $G m \mathrm{QUAC1}{ }^{\mathrm{NaCl}}$ or $\mathrm{GmQUAC} 1$ malate (purified in $150 \mathrm{mM} \mathrm{NaCl}$ or $75 \mathrm{mM} \mathrm{L}$ malate, respectively) were added to the cis-side. The trans-chamber, representing the extracellular compartment, was connected to the head stage input of a bilayer voltage-clamp amplifier. The cis-chamber, representing the cytoplasmic compartment, was held at virtual ground.

b, Representative current traces for single channel analysis of $\mathrm{GmQUAC} 1^{\mathrm{NaCl}}$ are shown at $+60 \mathrm{mV}$ or $-60 \mathrm{mV}$ holding potentials in the $\mathrm{NaNO}{ }_{3}$ solution. The closed (C) and full-open $(\mathrm{O})$ states are indicated.

c and d, The current-voltage relationships (c) and open probabilities (d) for the single-channel recordings of GmQUAC1. The chamber solutions $\left(\mathrm{NaNO}_{3}\right.$ or $\left.\mathrm{NaCl}\right)$ and protein $\left(G m \mathrm{QUAC} 1^{\mathrm{NaCl}}\right.$ or $\left.G m \mathrm{QUAC} 1^{\text {malate }}\right)$ are indicated in the inset (Data are mean $\left.\pm \mathrm{SEM}, \mathrm{n} \geq 4\right)$. 
a
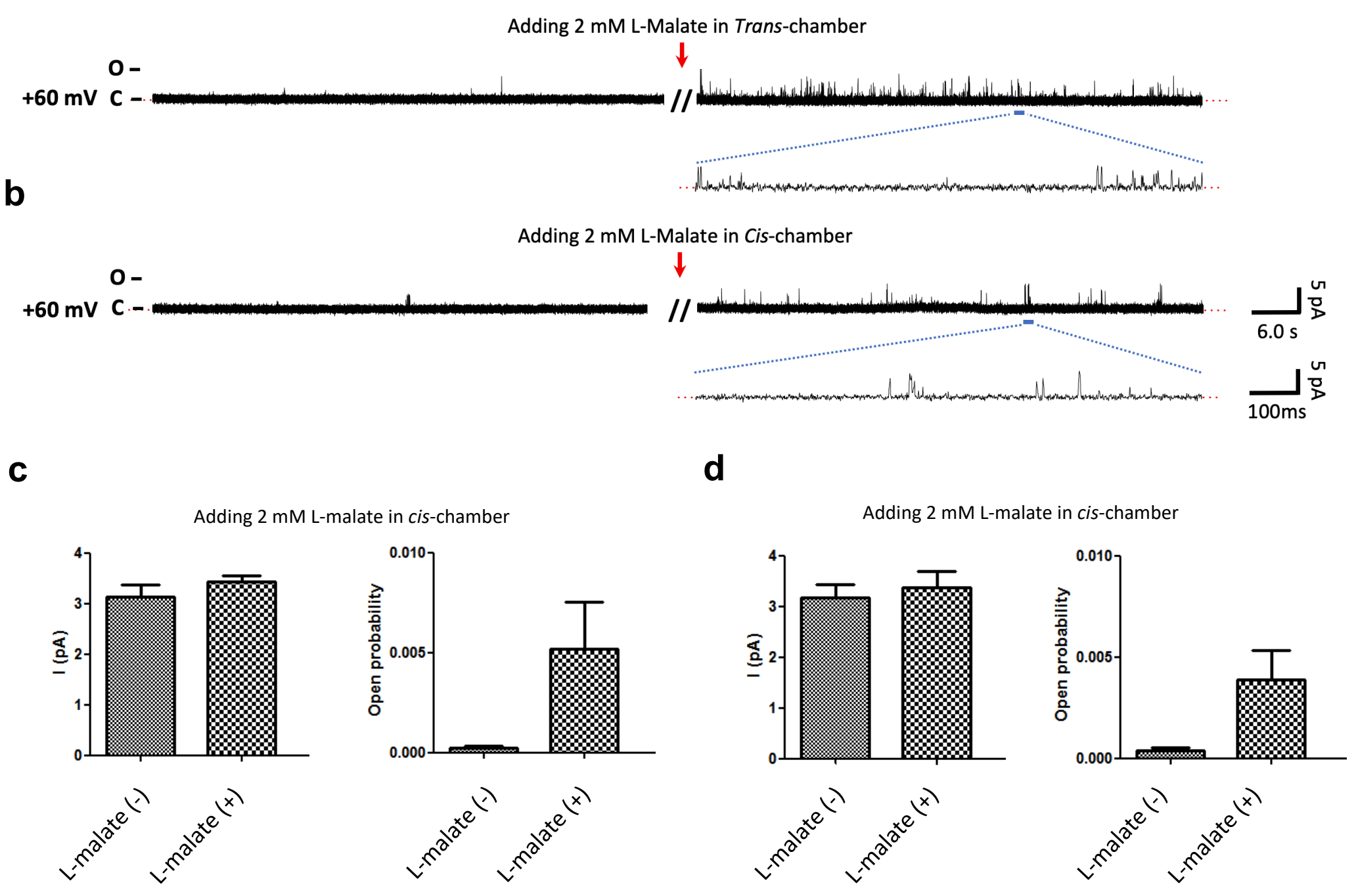

Fig. 6 Single channel analysis of malate regulation on QUAC1

$\mathbf{a}$ and $\mathbf{b}$, The chambers were filled with symmetrical solutions of $150 \mathrm{mM} \mathrm{NaCl}$, and the purified $\mathrm{GmQUAC1} 1^{\mathrm{NaCl}}$ proteins were added to the cis-side. The representative current traces of $60 \mathrm{~s}$ at $+60 \mathrm{mV}$, before and after adding $2 \mathrm{mM} \mathrm{L-malate}$ to the trans- (a) or cis- (b) chamber, are shown.

c and d, The regulation effect of malate on the $\mathrm{GmQUACl}{ }^{\mathrm{NaCl}}$ in the $\mathrm{NaCl}$ solutions. The current amplitude (left) and open probability (right) analysis for the recordings, before (-) or after (+) addition of $2 \mathrm{mM} \mathrm{L-malate} \mathrm{to} \mathrm{the} \mathrm{trans-} \mathrm{(c)} \mathrm{or} \mathrm{cis-} \mathrm{(d)} \mathrm{chamber}$ (Data are mean $\pm \mathrm{SEM}, \mathrm{n}=3$ for each group). 
a

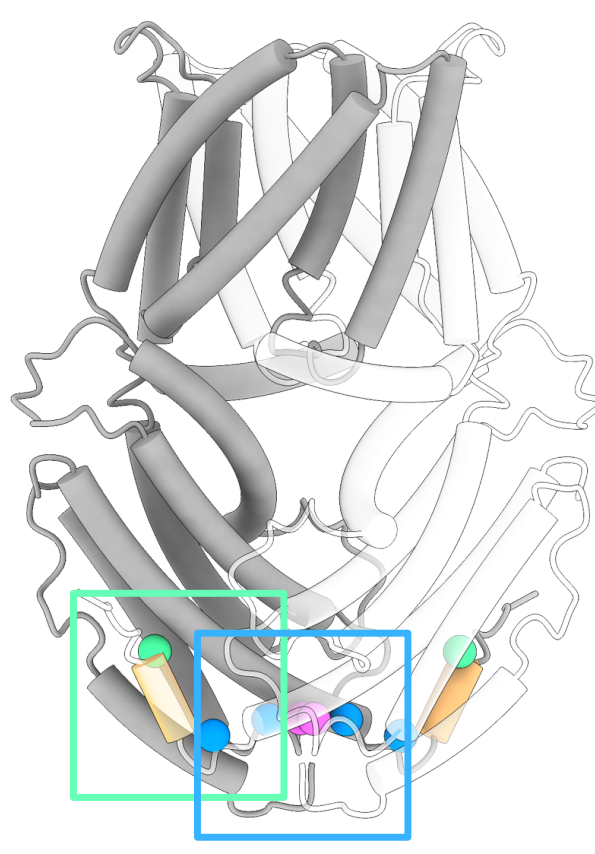

d

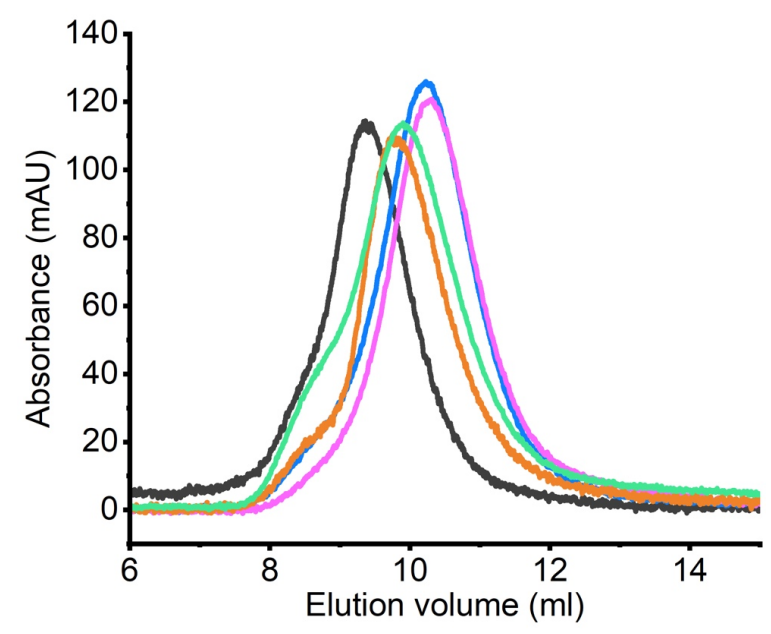

b

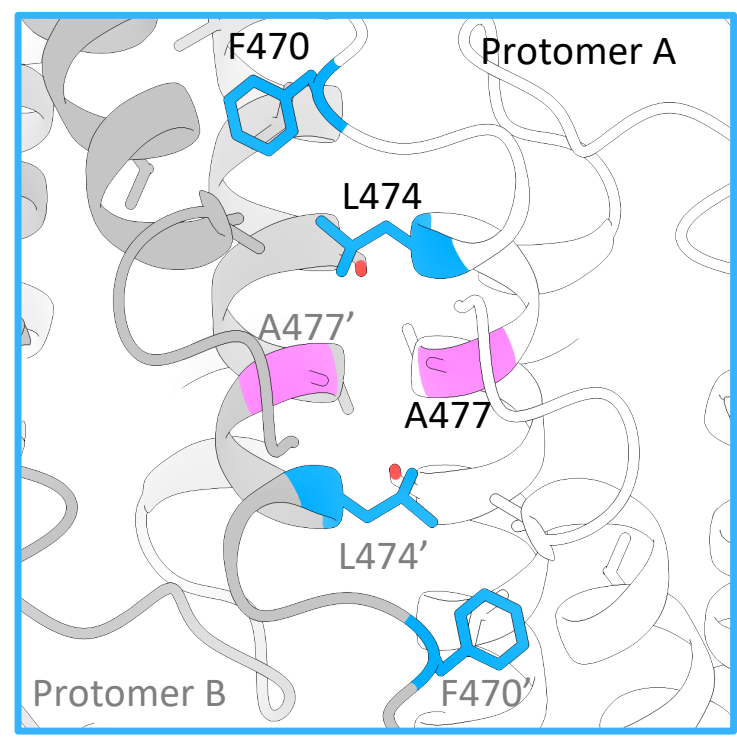

e

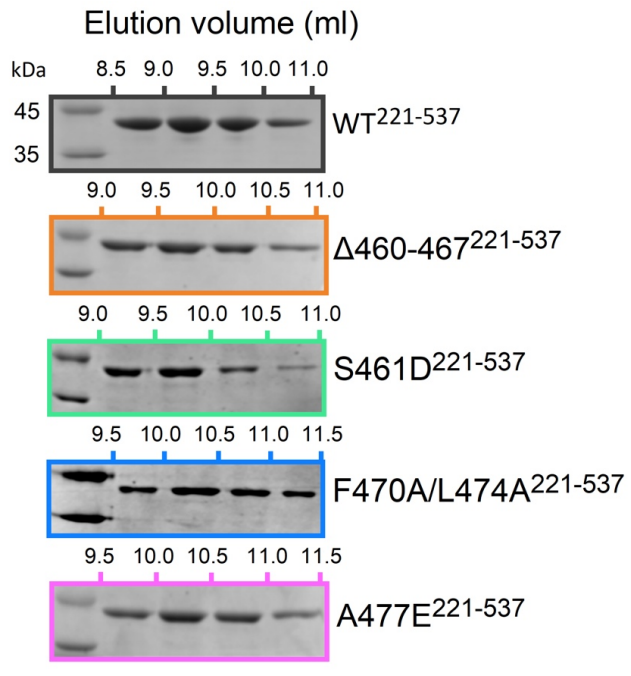

C

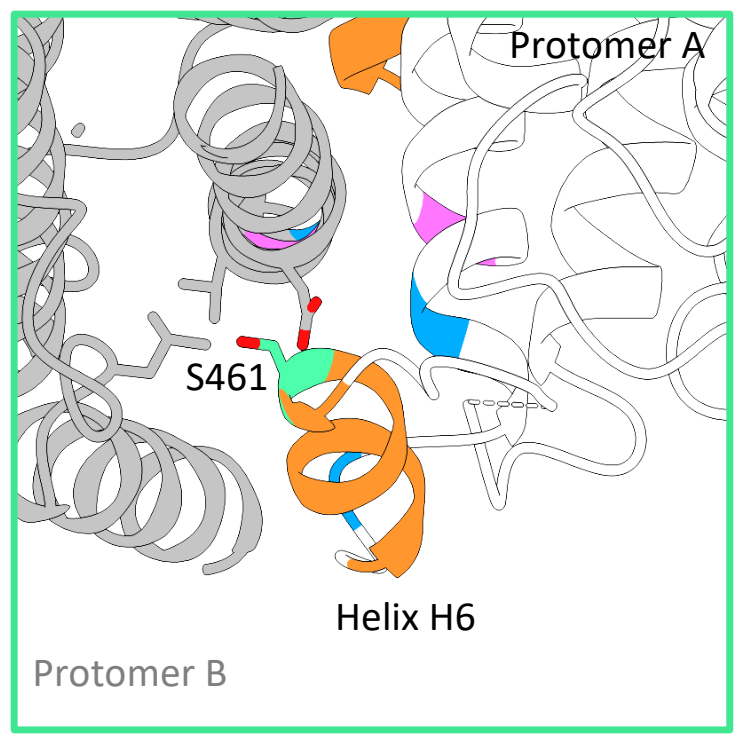

$\mathbf{f}$

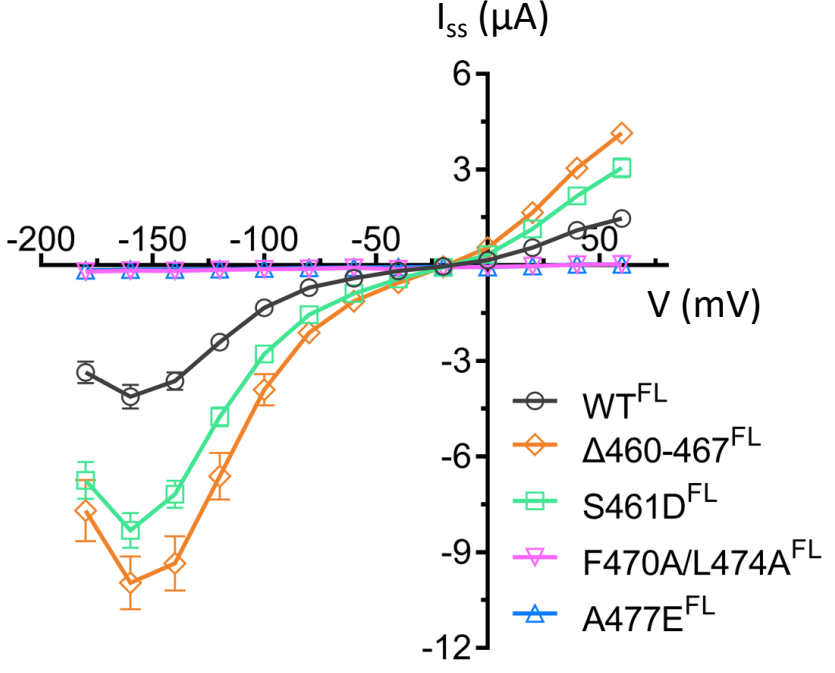

g

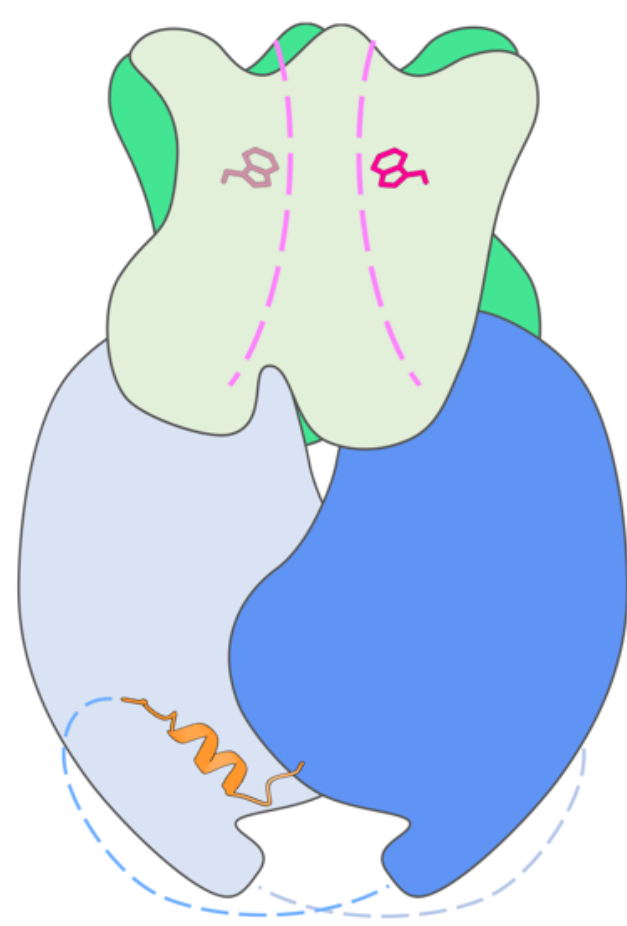

Closed
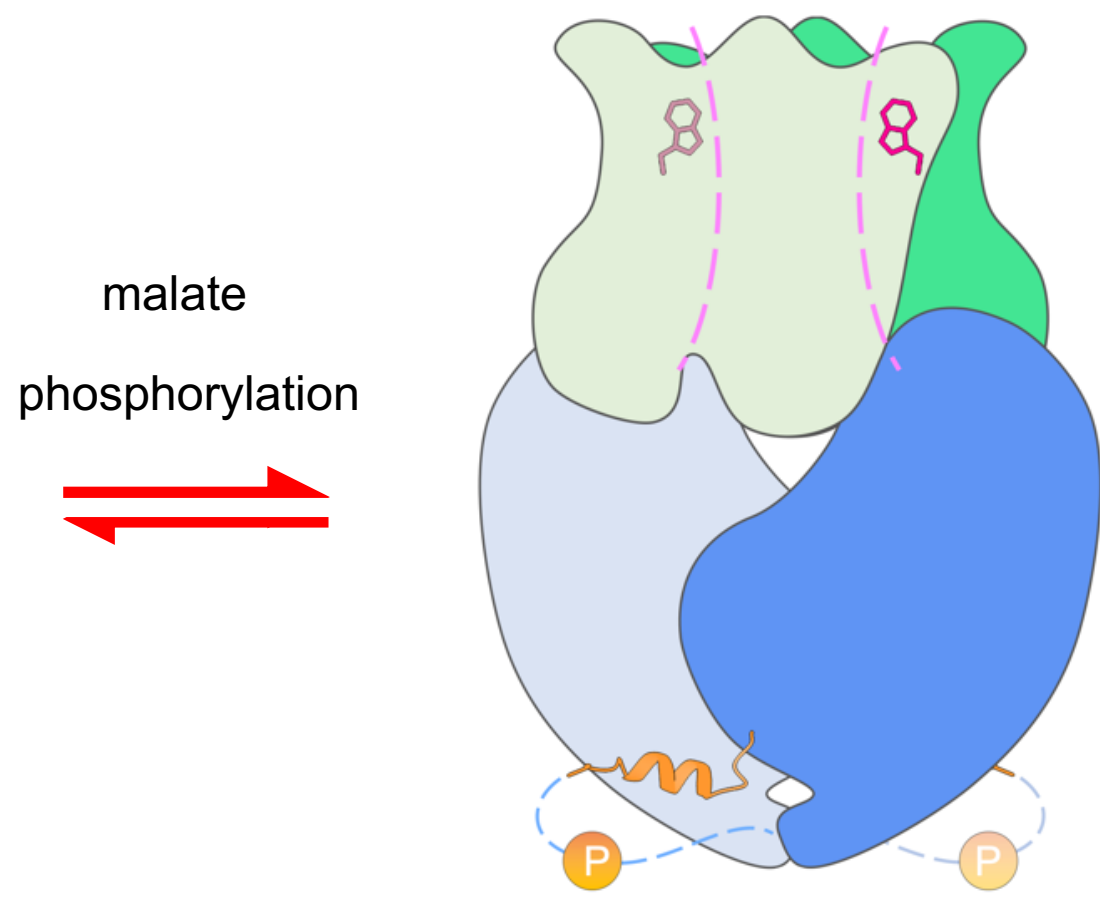

Open 
a, The cartoon of the GmQUAC1 channel is shown, with one protomer in grey and one in white. The domain-swapped helix (H6, residues 460-467) is colored in orange, and key residues at the dimeric interface are highlighted: S461 in green, A477 in magenta and F470/L474 in blue.

b and c, Close-up views of the cytoplasmic dimer interface. Residues at the dimeric interface are shown as sticks and colored as in (a).

$\mathbf{d}$ and $\mathbf{e}$, The purification of the CHD proteins (residues 221-537, wild-type or mutants) on a Superdex75 (10/300) column. Compared to the wild-type (peak at $\sim 9.2 \mathrm{ml}$ ), the eluted peaks for the dimer-disrupting mutants (A477E and F470A/L474A) shift backwards to $\sim 10.2$ $\mathrm{ml}$, suggesting a disassociation of dimer into monomer. The elution peaks at $\sim 9.7 \mathrm{ml}$ for the two finger helix altered mutants $(\Delta 460-467$ and S461D) suggest loosened conformations upon the removal of finger helix interaction. The eluted fractions were also analyzed by SDS-PAGE, as shown in (e).

f, The TEVC recording of GmQUAC1 channel (wild-type and mutants) in Xenopus laevis oocytes. The same set of mutations, as in (d), were generated into full-length constructs for conductance measurement in the bath solution of $30 \mathrm{mM}$ L-malate (Data are mean $\pm \mathrm{SEM}$, $\mathrm{n} \geq 8$ ).

g, Model for the QUAC1 channel regulation. Upon malate binding, the QUAC1 undergoes intramolecular domain reorganization to form a twisted bi-layer architecture, and subsequently induces the conformational change of the toggle switch W90. These changes promote the channel conversion from closed to open state. Other modulations, such as phosphorylation or calmodulin binding, can further enhance the malate-mediated activation, probably via the release of its inhibitory domain-swapped helix from the inter-protomer interaction. 